\title{
Architectures and Mechanical Properties of Drugs and Complexes of Surface-Active Compounds at Air-Water and Oil-Water Interfaces
}

\author{
Dipak K. Sarker* \\ Interfacial Nanotechnology Group, School of Pharmacy and Biomolecular Sciences, The University \\ of Brighton, Moulsecoomb Campus, Lewes Road, Brighton, BN2 4GJ, UK
}

\begin{abstract}
Background: Drugs can represent a multitude of compounds from proteins and peptides, such as growth hormones and insulin and on to simple organic molecules such as flurbiprofen, ibuprofen and lidocaine. Given the chemical nature of these compounds two features are always present. A portion or portions of the molecule that has little affinity for apolar surfaces and media and on the contrary a series of part or one large part that has considerable affinity for hydrophilic, polar or charged media and surfaces. A series of techniques are routinely used to probe the molecular interactions that can arise between components, such as the drug, a range of surface-active excipients and flavor compounds, for example terpenoids and the solvent or dispersion medium.
\end{abstract}

Results: Fifty-eight papers were included in the review, a large number (16) being of theoretical nature and an equally large number (14) directly pertaining to medicine and pharmacy; alongside experimental data and phenomenological modelling. The review therefore simultaneously represents an amalgam of review article and research paper with routinely used or established (10) and wellreported methodologies (also included in the citations within the review). Experimental data included from various sources as diverse as foam microconductivity, interferometric measurements of surface adsorbates and laser fluorescence spectroscopy (FRAP) are used to indicate the complexity and utility of foams and surface soft matter structures for a range of purposes but specifically, here for encapsulation and incorporation of therapeutics actives (pharmaceutical molecules, vaccines and excipients used in medicaments). Techniques such as interfacial tensiometry, interfacial rheology (viscosity, elasticity and viscoelasticity) and nanoparticle particle size (hydrodynamic diameter) and charge measurements (zeta potential), in addition to atomic force and scanning electron microscopy have proven to be very useful in understanding how such elemental components combine, link or replace one another (competitive displacement). They have also proven to be both beneficial and worthwhile in the sense of quantifying the unseen actions and interplay of adsorbed molecules and the macroscopic effects, such as froth formation, creaming or sedimentation that can occur as a result of these interactions.

\footnotetext{
*Address correspondence to this author at the Interfacial Nanotechnology Group, School of Pharmacy and Biomolecular Sciences, The University of Brighton, Moulsecoomb Campus, Lewes Road, Brighton, BN2 4GJ, UK; Tel: +44 (0)1273 641779; Fax: +44 (0)1273 642674; Email: d.k.sarker@brighton.ac.uk
} 
Conclusion: The disclosures and evaluations presented in this review confirm the importance of a theoretical understanding of a complex model of the molecular interactions, network and present a framework for the understanding of really very complex physical forms. Future therapeutic developer rely on an understanding of such complexity to garner a route to a more successful administration and formulation of a new generation of therapeutic delivery systems for use in medicine.

Keywords: Adsorbed layer, crosslinking, surfactant, polymer, drug, surface-active compounds.

\section{INTRODUCTION}

Many pharmaceutical and biomedical products, such as sterile injections of therapeutic proteins, simple (colloids) and complex vaccines (multiphase emulsions) and standard prescription drugs and over-the-counter (OTC) medicines, such as anti-dermatitis shampoos, creams, lotions and ointments, liposomal dispersions and coarse dispersions of solids are based on the use of wetting agents and hydrocolloid stabilizers or thickening agents $[1,2]$. The wetting agents are typically simple non-ionic surfactants (emulsifiers), such as polysorbates (Tweens), sugar esters (Spans), chemically-modified celluloses and blocked copolymers (Pluronics and Tetronics) but are also often low molecular weight natural charged species, such as fatty acids and lecithins (phosphatidylcholines) [3]. In addition to the functional formulation aids or excipients, such as, emulsifiers, other common ingredients in pharmaceutical and therapeutic formulations include the active pharmaceutical ingredient (API; active; drug) itself and shelf life maintaining agents that secure the integrity of the therapeutic, such as isotonicity salts and sugars, buffers, sequestrants, antioxidants and acidifiers [1]. Vaccines represent particularly complex forms of medicines as they often contain solids, such as alum (aluminum nanoparticles), emulsions, cell membrane lipids, traces of culture media, cellular fragments and proteins. Key ingredients in vaccines, course dispersions (suspensions of solids, foams, emulsions) and colloidal therapeutics (polymer micelles and liposomes) are indicated in Table 1. Such a complex array of ingredients (excipients) are known as adjuvants in a vaccine and used to promote a larger immune response upon injection into the blood [2]. Both egg and milk proteins are found in significant concentrations within many vaccines, such as the diphtheria, acellular pertussis and tetanus (DaPT) vaccine.

Simple drug types include, antibiotics (e.g. erythromycin, ciprofloxacin), anesthetics (e.g. li- docaine, propofol), cell destroying - cytotoxics (e.g. paclitaxel, doxorubicin, methotrexate), antiinflammatory drugs (e.g. betamethasone, flopropione), hormones (e.g. estradiol, insulin), psychoactive API's (e.g. lithium carbonate, amitriptyline), and many more formulated intro therapeutic products. The range of forms observed is large and traverses simple solutions and colloids [4-6], to products based on fibers $[7,8]$. In order to understand the working of such complex systems, it has been necessary to start by looking at simple models of the interplay [9] between polymers (polysaccharides, gums, complex lipids, emulsifiers, proteins and oligonucleotides), organic molecules and excipients [10,11]. A list of common vaccine, food and pharmaceutical surface-active agents and product ingredients is presented in Table 2 . The simple active species (exception of insulin), are not presented in the Table but are mentioned at relevant points in this review. Many of these therapeutics possess a high octanol-water partition coefficients (as hydrophobes) or $\log P$ values, and consequently show varying degrees of surfaceactivity. In addition to the formal pharmaceuticals, many novel and emerging products with "supposed" therapeutic properties used in "nutraceutical' (nutritional-pharmaceuticals e.g. hydrophobic hop iso-acids and vitaminized supplements) and 'cosmeceutical' (cosmetic-pharmaceuticals e.g. glycosylated-polyunsaturated lipid) formulations [12] also show significant excipient-interaction properties and surface-activity [13]. These products often contain polysaccharides [14] with or without flavanols, quinones and polyphenolic compounds, such as ferulic acid, quercetin, catechin, curcumin and phloroglucinol $[11,15]$.

Biomedical applications of API and excipients $[4,10,16,17]$ extend to coatings for surgical devices and other drug eluting devices, such as transdermal (patch) and subcutaneous implants (scaffolds). Here, modified surfaces and hydrophilization using surfactant coverage can aid wettability. This type of wetting is often used to hydrophilize surfaces and 
Table 1. Indication of the components routinely found in medicines and therapeutics, such as vaccines, coarse dispersions and nanomedicines.

\begin{tabular}{|c|c|}
\hline Ingredient/Excipient in Product & Role \\
\hline Egg proteins from culture medium & Vaccine component \\
\hline \multicolumn{2}{|l|}{ Ovalbumin } \\
\hline \multicolumn{2}{|l|}{ Conalbumin } \\
\hline Milk proteins from culture medium & Vaccine component \\
\hline \multicolumn{2}{|c|}{$\beta$-casein and caseinates } \\
\hline \multicolumn{2}{|c|}{$\alpha$-lactabumin } \\
\hline \multicolumn{2}{|c|}{ Bovine serum albumin (BSA)* } \\
\hline Bacterial and yeast proteins & Vaccine component from passaging $\dagger$ \\
\hline Human, animal and insect proteins & Vaccine component from passaging $\dagger$ \\
\hline Gelatin (collagen) & Vaccine and pharmaceutical component \\
\hline Gums and polysaccharides & Vaccine and pharmaceutical component \\
\hline Proteins and enzymes & Vaccine and pharmaceutical component \\
\hline Polysorbates (Tweens) and surfactants & Vaccine and pharmaceutical component \\
\hline Antibiotics & Vaccine and pharmaceutical component \\
\hline Silica and silicates & Pharmaceutical component \\
\hline Latexes & Pharmaceutical component \\
\hline Alum (potassium aluminium sulphate) & Vaccine component \\
\hline Microbial antigens (cell fragments) & Vaccine component \\
\hline Natural (animal/plant) and synthetic oils & Vaccine and pharmaceutical component \\
\hline
\end{tabular}

Vaccines contain a minimum on average $\sim 2 \mu \mathrm{g} / \mathrm{ml}$ proteins e.g. ovalbumin or caseinates from culture medium or the cultured cell. The content can be considerably higher.

*HSA the human version of BSA is used in a number of injectable pharmaceuticals to aid long-circulation in the blood $e . g$. Abraxane ${ }^{\circledR}$ (a 130 nm paclitaxel-HSA nanoparticle conjugate used for breast cancer treatment).

Passaging or sub-culturing refers to the practice of transferring cells from an original culture to fresh growth medium to maintain the pathogen culture and expand the number of cells it is also a means of assisting the loss of virulence of a pathogenic strain.

Table 2. Surface active materials described in the figures of the article and used ubiquitously in foods and medical products.

\begin{tabular}{|c|c|c|}
\hline Surface Active Agent & Source & Molecular Weight \\
\hline \hline Bifunctional agents & & \\
\hline Arabinoxylan (ferulate ester) $\dagger$ & Rye & Average $0.6 \mathrm{MDa}(370-870 \mathrm{kDa})$ \\
\hline$(+)$-catechin (flavan-3-ol) & Plant material & $290 \mathrm{Da}$ \\
\hline Isohumulone & Hops & $362 \mathrm{Da}$ \\
\hline Pectin (polygalacturonic acid) & Fruit & Average $95 \mathrm{kDa}$ \\
\hline Methylcellulose $(\mathrm{MeC})$ & Semi-synthetic & Average $145 \mathrm{kDa}$ \\
\hline Proteins & & \\
\hline
\end{tabular}




\begin{tabular}{|c|c|c|}
\hline Surface Active Agent & Source & Molecular Weight \\
\hline$\beta$-casein & Bovine milk & $24 \mathrm{kDa}$ (phosphorylated protein) \\
\hline$\alpha$-lactalbumin & Bovine milk & $14 \mathrm{kDa}$ (globular protein) \\
\hline$\beta$-lactoglobulin & Bovine milk & $18.6 \mathrm{kDa}$ (globular protein) \\
\hline Ovalbumin & Chicken egg & $43 \mathrm{kDa}$ (glycoprotein) \\
\hline Insulin protein & Bovine pancreas & $5.8 \mathrm{kDa}$ (twin-chained peptide) \\
\hline \multicolumn{3}{|l|}{ Simple surfactants } \\
\hline Pluronic F108 (poloxamer 338) & Synthetic & $14.6 \mathrm{kDa}(80 \%$ hydrophile $)$ \\
\hline ODAF - 5-N-(octadecanoyl) aminofluorescein & Synthetic & $614 \mathrm{Da}$ (fluorescent surfactant) \\
\hline Tween 20 - polyoxyethylene(20)-sorbitan monolaurate & Synthetic & $1224 \mathrm{Da}$ (non-ionic surfactant) \\
\hline LPC - L- $\alpha$-lysophosphatidylcholine, monopalmitate: monostearate 2:1 (lyso-lecithin from egg) & Chicken egg & $496 \mathrm{Da}$ (zwitterionic surfactant) \\
\hline Di-palmitoyl phosphatidylcholine (DPPC) & Soya & $734 \mathrm{Da}$ (zwitterionic lipid) \\
\hline Cholesterol & Chicken egg & $387 \mathrm{Da}$ (sterol, bile precursor) \\
\hline
\end{tabular}

$\dagger$ - Arabinoxylan is also known as pentosan.

\$ - ODAF (or equivalent fluorophore) is not used in a therapeutic context but is a fluorescent probe molecule used for measurements of molecular mobility.

'bind' a layer of water in particles and prevent them from developing biofilms in situ or being removed $[11,17]$ from the blood (in parenteral injected products), via a complex biochemical process of labelling with blood proteins and antibodies (opsonization) and then engulfment by blood phagocyte cells (white blood cells; macrophages) as part of the body's defenses $[2,11]$. More general colloid science and medical nanotechnology involve more familiar drug delivery vehicles [8], these being micelles, liposomes, solid lipid nanoparticles (solid emulsions; SLNs), nanogels (polymer micelles) and molecular drug-polymer conjugates [6, 11], known as pro-drugs. Encapsulation of therapeutic species in these dispersion forms is frequently used for confinement of colloidal isotopes for chemotherapy and radiotherapy purposes and CAT scan or MRI imaging aids, such as elemental gadolinium [1,2].

\section{UNDERSTANDING SYSTEM BEHAVIOR: MODELS}

A number of groups have studied the properties of polymers [18-22] and surfactants/emulsifiers $[10,23,24]$ at macroscopic surfaces, and this re- mains the reasonable starting point for a better understanding of the behavior of polymer, actives and surfactants (formulating aids) in medical and pharmaceutical dispersions. A particularly useful starting point for looking at therapeutic dispersion behavior and commercial-stability, therefore efficacy, has been to consider the behavior of protein (usually globular proteins) at air-water $(\mathrm{A} / \mathrm{W})$ and oil-water $(\mathrm{O} / \mathrm{W})$ surfaces or interfaces, respectively $[25,26]$. Globular proteins, such a bovine serum albumin (BSA), human serum albumin (HSA) and $\beta$-lactoglobulin $(\beta L G)$, are widely studied across the board [27-33] by colloid scientists by virtue of their compact size, near ideal profile of the four classes of amino acids (aromatic-aliphatic, acidicbasic, sulfur-containing and polar), superb surfaceactive properties, non-toxic nature and ease of isolation from a biological source.

The reasoning behind their ubiquitous use, is two-fold. First, proteins represent nearly-ideal models of strongly-interacting polymers because of their capability to form ionic, covalent, apolar, hydrogen-bond and disulfide bridge formal bonds and interactions. Second, they have biological and 
medical relevance because in the period 20052017 more than $25 \%$ of all currently licensed, new and in-development therapeutics are based on proteins and antibodies (proteins). This figure is estimated to top the $50 \%$ mark some decades into the future $[1,2]$. A large number of colloid and surface groups traversing those involved with theoretical modelling $[19,20,23,34]$ and more pragmatic practical understanding $[12,30,35-37]$ have looked at the properties of proteins at $\mathrm{A} / \mathrm{W}$ and $\mathrm{O} / \mathrm{W}$ interfaces. Areas of study of the mechanical and physico-chemical properties of adsorbed protein films have involved biophysics [38], medicine $[7,9,39]$, food $[14,30]$ and agricultural applications. However, because of their molecular mass, which can be anything from a small, $5800 \mathrm{Da}$ (insulin) to for example, a large, severe acute respiratory virus coronavirus accessory protein-7a (SARS virus protein) of $201000 \mathrm{Da}$, this means diffusion to and re-configuration at the $\mathrm{A} / \mathrm{W}$ or $\mathrm{O} / \mathrm{W}$ interface can be lengthy, unlike a simple low molecular weight surfactant $[3,5,27,40,41]$, which show rapid surface diffusion, such as Tween 20 .

\subsection{Surface Tension and Adsorption to the In- terface}

A convenient way of understanding the basics of protein adsorption at the interface is by evaluating the ease by which and time involved to achieve equilibrium surface tension values. A/W measurement are generally more convenient than $\mathrm{O} / \mathrm{W}$ measurements using many forms of modern apparatus [28, 32, 34, 40, 41] and frequently having yielded similar data. In some approximate way, $\mathrm{A} / \mathrm{W}$ measurements and $\mathrm{O} / \mathrm{W}$ measurements double-up as 'air' can be viewed as a loose model of the oil-phase of emulsion by virtue of its dielectric constant, which is about half of most oils and nearly 80 times less than water [2] but with this distinct concept, as has always been soundly argued, lacking the increased density and viscosity may inhibit species inter-penetration into the oil-phase. Recent studies have however indicated that liquid hydrocarbons may allow surfactant-like species considerable freedom to enter the oil phase unlike the gel phase, as fats used in SLNs [19, 26, 28, 33, 34] may constrain movement due to impenetrability.

The equilibrium or near-equilibrium surface tension values for a series of proteins of food and pharmaceutical use are shown in Fig. 1 (Part A).
As in the case of many proteins for the protein shown, the rate of diffusion to the interface and species equilibrium attainment is based on the physico-chemical properties [33], functional group chemistry of the protein and extrinsic environmental properties, such as mechanical denaturing, temperature and oxygen tension of the gas and liquid phases [40]. The cartoon associated with Fig. 1 shows the simplified process, studied by many with which proteins adhere to the interface [18, 42]. The three fundamental steps shown in Part B are diffusion to the interface and surface tension lowering, re-arrangement and interaction, with the last two taking place congruously [3]. Phosphorylated proteins, such as highly hydrophilic $\beta$-casein and glycoproteins such as, conalbumin (ovotransferrin) and ovalbumin are particularly good 'surface-active polymers' lowering surface tension to less than $52 \mathrm{mN} / \mathrm{m}$ at $20^{\circ} \mathrm{C}$. Ovotransferrin also serves as an anti-microbial agent because of its ability to complex metal ions and thus, deprive the microbe of metabolic nutrients and in this manner doubles as a therapeutic. Once adsorbed at the interface, inter-molecular interactions for the protein molecule of the type indicated in Fig. 2, such as ion-pair formation and entanglement [26, 28, 4345] and evidence of species linking, can serve to modify the mechanical properties of the adsorbed layer and its architecture.

\subsection{Weak and Strong Interaction}

The structure of the adsorbed layer formed at A/W and $\mathrm{O} / \mathrm{W}$ interfaces described in pictorial form in Fig. 2 is shown in quantitative graphical form in Fig. 3 (Part A), described by surface tension values, for a series of proteins, methyl cellulose $(\mathrm{MeC})$ and low molecular weight non-ionic surfactant, Tween 20. The figure shows proteins, Tween 20 and $\mathrm{MeC}$ alone but also in combination, where either the concentration of the $\mathrm{MeC}$ is described or the molar ratio $(R)$ of small molecule surfactant to protein helps to describe bulk compositional effects on surface dominance and composition [5, $19,22,30,38,46]$ in terms of surface prevalence. Simple solutions containing colloidal protein, or polymer have characteristic equilibrium surface tensions, which chart an average surface composition. On a concentration basis, conalbumin shows the lowest equilibrium surface tension values, this indicates a dense surface coverage and a strongly interacting surface adsorbed layer, represented in the schematic cartoon also in Fig. 3 (Part B). 
Part A

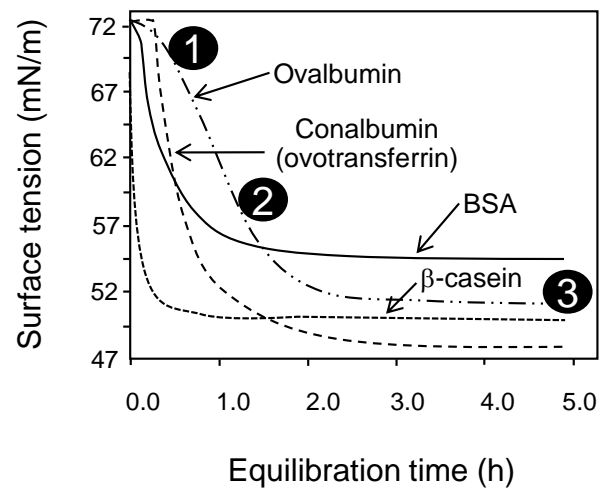

Part B

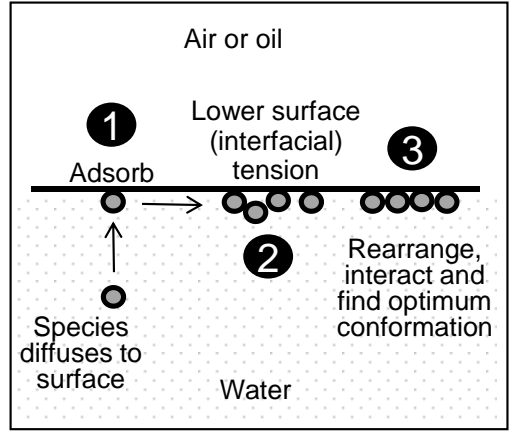

Fig. (1). Surface diffusion and adsorption equilibration plot for four globular proteins (arrowed in the plot), as models for species with the potential for extensive intermolecular interactions (Part A) using surface tension measurement. Phenomenological representation of the diffusion to, adsorption at, and rearrangement of highly surfaceactive polymeric surfactants at a hypothetical surface (Part B). All solutions are made in $0.05 \mathrm{M}$ phosphate buffer, pH 7.0 unless otherwise stated.

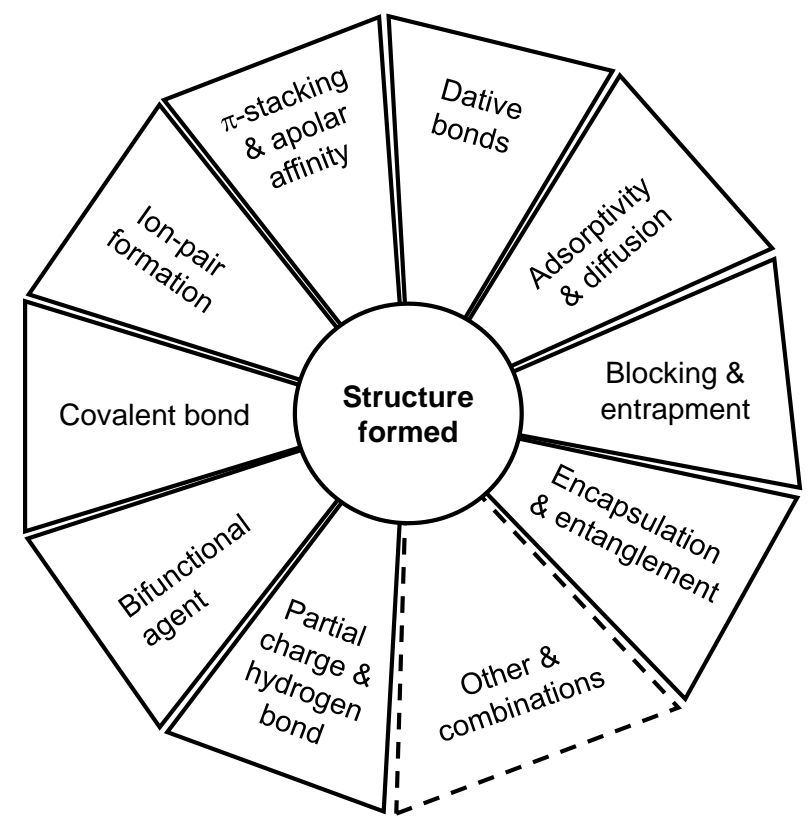

Fig. (2). Representation of the scheme of intra- and inter-molecular interactions for a range of surface active species such as, complexes with surface activity, classic small molecule surfactants, polymeric surfactants, surface active drugs and proteins.

The Fig. Part A, also shows in phenomenological form the surface coverage by Tween 20 alone, which at approximately $12 \mu \mathrm{M}$ is about $33 \mathrm{mN} / \mathrm{m}$, like conalbumin. Proteins and surfactants do not form the same type of surface architectures [3, 41]. The essential difference between conalbumin and the Tween 20 lies in the breadth and magnitude of inter-molecular interactions. With the globular proteins these are extensive, so as to yield a strongly-interacted surface layer, whilst with Tween 20 (an example of a simple surfactant) lateral interaction between adjacent molecules is weaker but the species itself is able to diffuse rapidly to repair portions of poor surface coverage, generated by thermal and mechanical perturbation [26, 34], maintaining an even surface coverage. Combining surface-active polymer, such as $\mathrm{MeC}$ [19] and BSA [29] means poorer surface tension than $\mathrm{MeC}$ alone at equivalent concentrations, thus pointing toward bulk binding or less effective surface coverage. With Tween 20 and $\beta$-casein the explanation is clearer and has been reported elsewhere [3, 40]. Here, interaction between the 24 $\mathrm{kDa}$ protein and $1.2 \mathrm{kDa}$ Tween may result in an association of the two that creates a more surfaceactive species than either of the two individual components $[35,38]$, without compromising interfacial loading and molecular packing.

\subsection{Small, Large and Hybrid Molecule Net- works}

Foams generated using $76 \mathrm{kDa}$ conalbumin and Tween 20 (Fig. 4, Part A) represent a macroscopic reflection of the interfacial behavior described on numerous occasions [27, 36, 41, 47] in foams and foam films in earlier work. Bubbles in the foam, which are covered by the protein (these can be considered as if they were also covered by a surface-active polymer that has scope for intermolecular interaction) in the form of a strongly 
Molar Ratio, $R$ (Tween 20:Protein)

$\begin{array}{lllllll}0.0 & 0.2 & 0.4 & 0.6 & 0.8 & 1.0 & 1.2\end{array}$

Concentration of all species other than $\mathrm{MeC}(\mu \mathrm{M})$

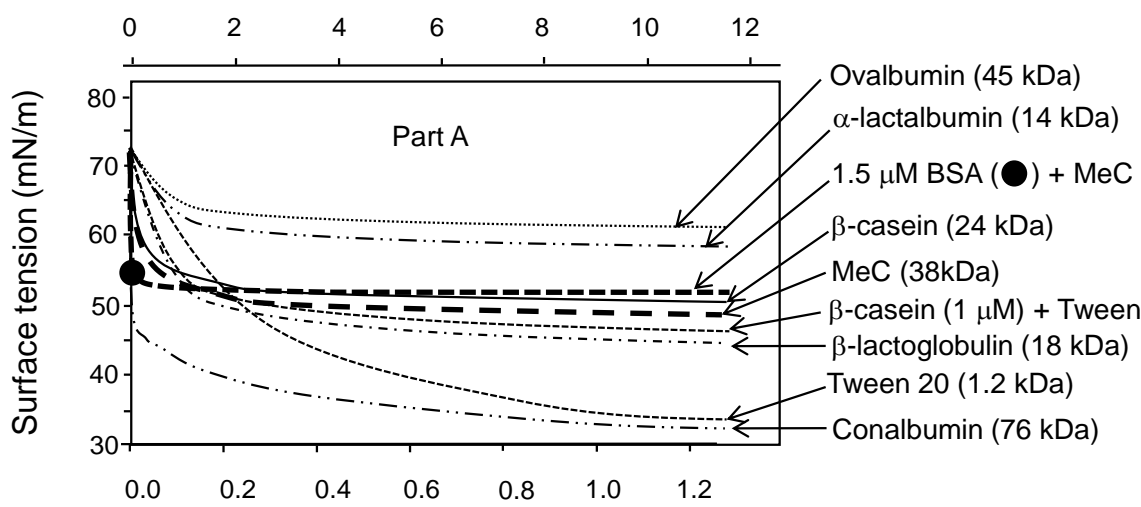

Part B

Air or oil

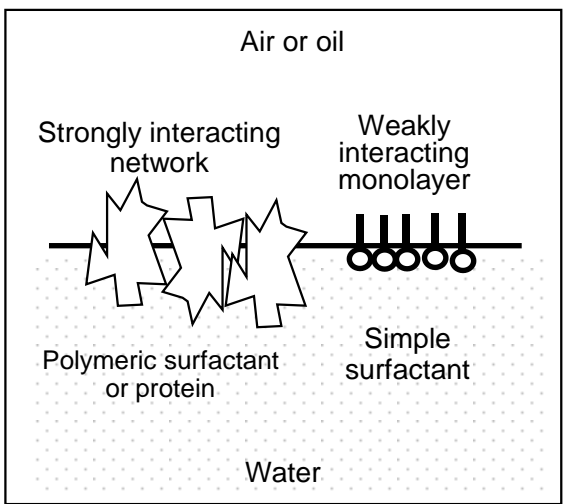

Methylcellulose concentration $(\mu \mathrm{M})$

Fig. (3). Surface tension lowering and structure forming capability of protein types as model interactive surfactants, for ovalbumin (OVA), $\alpha$-lactabumin ( $\alpha \mathrm{LA}$ ), bovine serum albumin (BSA), $\beta$-casein ( $\beta \mathrm{CAS}$ ), $\beta$-lactoglobulin $(\beta \mathrm{LG})$ and egg conabumin (CON) are shown, and simple non-ionic surfactant, Tween 20 (T20) and methylcellulose (MeC) (arrowed in the plot) are presented. Pure samples, such as pure BSA $(\mathbf{O})$ and mixtures of protein and simple surfactant or protein and cellulose derivative are indicated (Part A). Phenomenological representation of the structures formed by polymers and simple small molecules are shown (Part B). A crossover in the data for BSA, $\mathrm{MeC}$ and $\mathrm{BSA}$ with $\mathrm{MeC}$ point to binding of the polymer by the BSA to create a more surface active species. All solutions are made in $0.05 \mathrm{M}$ phosphate buffer, $\mathrm{pH} 7.0$ unless otherwise stated.

cohesive structured film. Whereas, bubbles fabricated from solutions containing simple low molecular weight surfactants have a thin and mechanically 'weak' surface coverage commensurate with the surfactant and steric restrictions on surface loading. Both these forms are shown pictorially in Fig. 4 (Part B). Interestingly, and studied on numerous occasions $[3,30,41]$, displacement of protein from the adsorbed layer, to be replaced by surfactant (Tween 20) compromises the water binding volume of the constituent foam cells and capacity for foam-entrained water. This has been attributed to variations of interfacial composition $[3,8,21$, $27,43]$ and the mechanical modifications that arise from surface compositional change. The consequences of such compositional variations, are differences in the loading of material at the interface and of palisade layer protrusion from the bubble (or droplet) surface into the continuous phase as pendant tails, loops and chains of the protein (or polymer), and associated water and steric repulsion between opposing faces of the foam film. This then makes the foam lamellae (thin liquid films) thinner and means the foam is less dense
[41]. Combinations of simple low molecular weight surfactant, protein/polymer and surfactantprotein or surfactant-polymer complexes with surface activity, and the interacted networks they are able to form, coexist in the plane of the interface [41].

\subsection{Competitive Adsorption and Displacement}

Fig. 4 shows in clear form two distinct principles. These are small molecule surfactants competing for space at the interface and displace those surface-active agents, in free form, which cannot reduce the surface tension down to the values typically associated with dense small molecule interfacial packing. This displacement in the case of the foams, represented in the Fig. (Part A), means a lower number of intact and thicker foam lamellae. This is usually, in foams, seen as bubble bursting and an increased tendency towards the disproportionation of bubbles as polymer molecules are displaced or small molecules preferentially adsorb in so-called "competitive displacement" or "competitive adsorption", respectively [3, 27, 41]. Alt- 


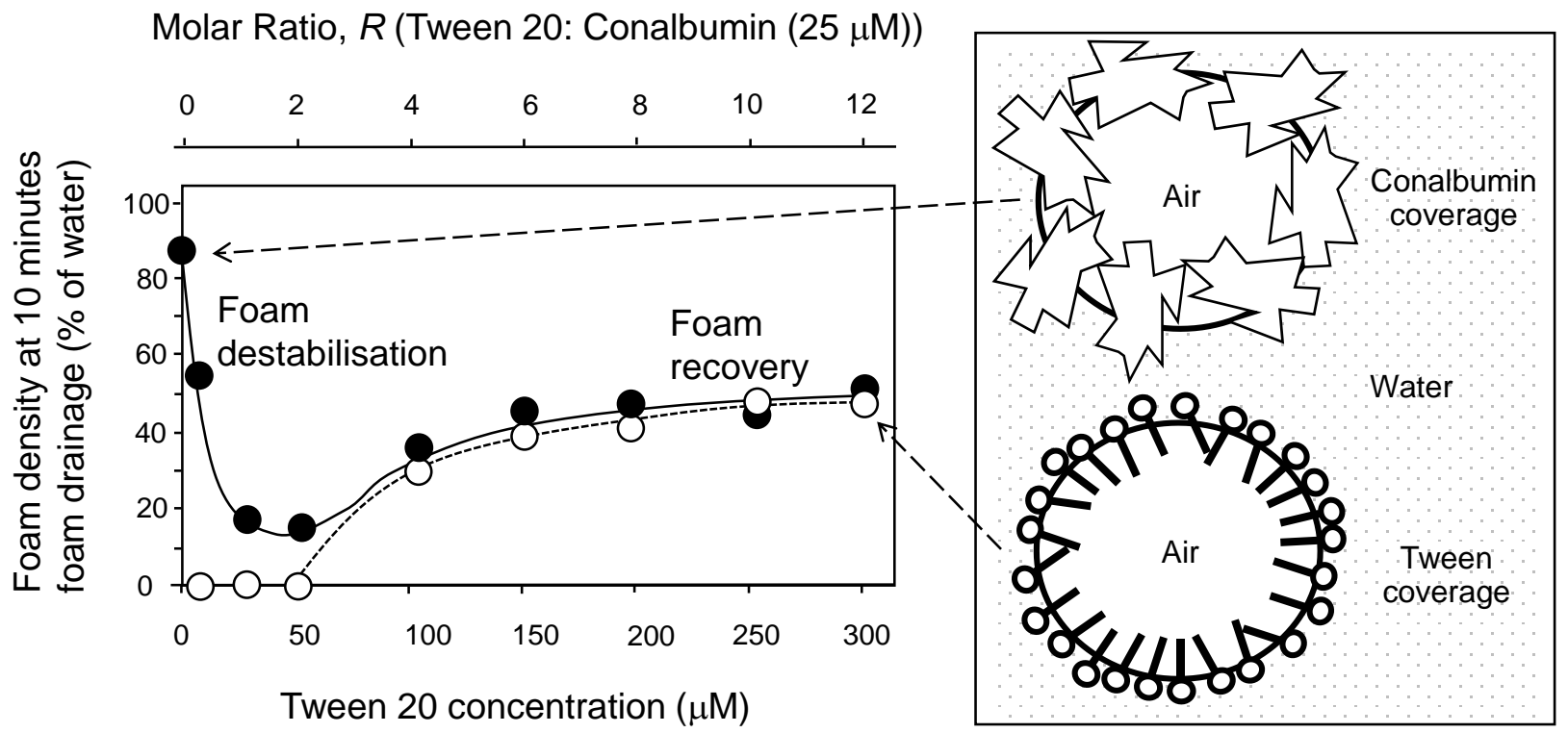

Fig. (4). Air-in-water or air/water (A/W) foams based on dilute dispersions of colloidal egg conalbumin, also known as ovotransferrin (O) in absence and the presence of increasing amounts of small molecule surfactant Tween 20 (polysorbate 20) and those formed entirely from Tween $20(\mathrm{O})$ are shown (part A). Phenomenological representation of the structures formed by polymers and simple small molecules on the surface of $<2 \mathrm{~mm}$ diameter bubbles (Part B), not drawn to scale. All solutions are made in $0.05 \mathrm{M}$ phosphate buffer, $\mathrm{pH} 7.0$ unless otherwise stated.

hough, unfortunate in foams, where proteins stabilize the gas cells within a foam product, such as egg protein foams or beer foam, this has provided a great opportunity in the biomedical field, for encapsulation of suitable 'therapeutic surfactants' in a near-whole polymeric-surfactant interfacial adsorbed layer (see section 6). Many therapeutic molecules are akin to surfactants, with lidocaine and amitriptyline being very obvious examples. Recent work has considered using this competitive adsorption phenomenon to 'house' API's on the surface of therapeutic shampoo foams and emulsions $[2,8,11]$.

\subsection{Molecular Rearrangement and Crosslink- ing}

The molecular re-arrangement associated with adsorption of amphiphiles at the surface of bubbles in foams and oil droplets (and pharmaceutical solids, such as solid particulate drugs) in coarse emulsions and nano-emulsions can be illustrated by data in Fig. 5 (Part A) involving an O/W interface and acting contrary to the irreversible adsorption described by Reichert and Walker [23] but illustrated clearly by peptide-layer 'switching' in the plane of the interface described elsewhere [26]. The Part shows the ease of adsorbed layer disruption using the sharp-edge of an interfacial rheometer bicone to the two-dimensional "gelled" adsorbed layer formed by $67 \mathrm{kDa}$ BSA, ovotransferrin and Tween20:BSA at a molar ratio of $R=0.9$. The plot shows how the network formed by both protein only systems, resists the effect of shearing at low rates of deformation, largely as a result of inter-molecular interactions and crosslinking of material adsorbed in the plane of the interface [28, $32,42]$, possibly compounded by the formation of multiple layers as seen with BSA on metal nanoparticles [29], and finally being broken at much higher shear rates $[34,48,49]$. The mix of protein and surfactant data is more complex in some sense, as initial deformation is permitted to much higher shear rates but at very high shear rate this appears to cause interfacial friction between "interfacial aggregates" and increase in measured shear stress. What is clear from the interfacial rheogram (Fig. 5, Part A) is that structure of proteins and polymers adsorbed at the interface is measura- 
ble and significant, as seen clearly in an article involving with galloylated quinic acid tannin-rich Tara gum and poly(vinyl alcohol) dispersions [50].

Fig. 5 (Part B) shows the storage $\left(G^{\prime}\right)$ and loss moduli $(G$ ' $)$ crudely analogous to adsorbed layer "elasticity" and "viscosity" in an A/W system. Similar rules apply and govern the behavior of adsorbed species, however, as can be seen by careful comparison with Part A. In Part B inter-molecular crosslinking between BSA yields high interfacial elasticity, as seen with gelation caused by laccase enzyme and the polyphenols of pectin [32] and augmented viscosity. $\beta$-casein has considerable interfacial elasticity, whilst ovotransferrin has a weaker adsorbed layer, these are plotted as a function of standing time, which is not dissimilar to the terminal values seen for a mixture of Tween 20 and BSA (based on added Tween 20 for measurements made after one hour equilibration), at a molar ratio, $R=0.9$. At this molar ratio, the network of interactions between BSA molecules is substantially compromised and disrupted (analogous to being dissolved), often meaning bubble rupture in foams, as comprehensive BSA intra-adsorbed layer interaction has been shown to safeguard bubble stability [29], and improve poor droplet coalescence resistance in emulsion droplets $[23,26,30]$.

\subsection{Networks and Architectures}

Proof of adsorbed layer interaction between the positive charges on the protein and those negative charges of carboxylic acid part-ionized poly(galacturonic acid), also known as pectin (Fig. 6, Part A) are supported by numerous reports of interfacial ion-pair formation [24, 44, 45]. Pectin use in pharmacy is on the increase given the biocompatible nature of the substrate, which is being used increasingly for gel and nanoparticle formation and this is likely to find further use in pharmacy in the future for a diverse range of surgical and medicinal products $[35,45]$. In a similar fashion to pectin, the protein crosslinking bifunctional agent, glutaraldehyde can be clearly seen in Fig. 6 (Part A) to aid linking of moieties with the manifestation of thicker foam films [27]. As a result of micro-molar inclusion of bifunctional agent, interfacial proteins in a part disrupted protein-Tween 20 system at molar ratio, $R=0.25$ are united to give better water-binding, and a larger intra-foam film disjoining pressure and interadsorbed layer repulsion to produce thicker foam films, as reported with oil droplet O/W films [19, 32]. Notably, adding too much bifunctional agent simply caused large-scale aggregation and aggregate formation, either destabilizing the film, possibly by causing local depletion of protein or pro-
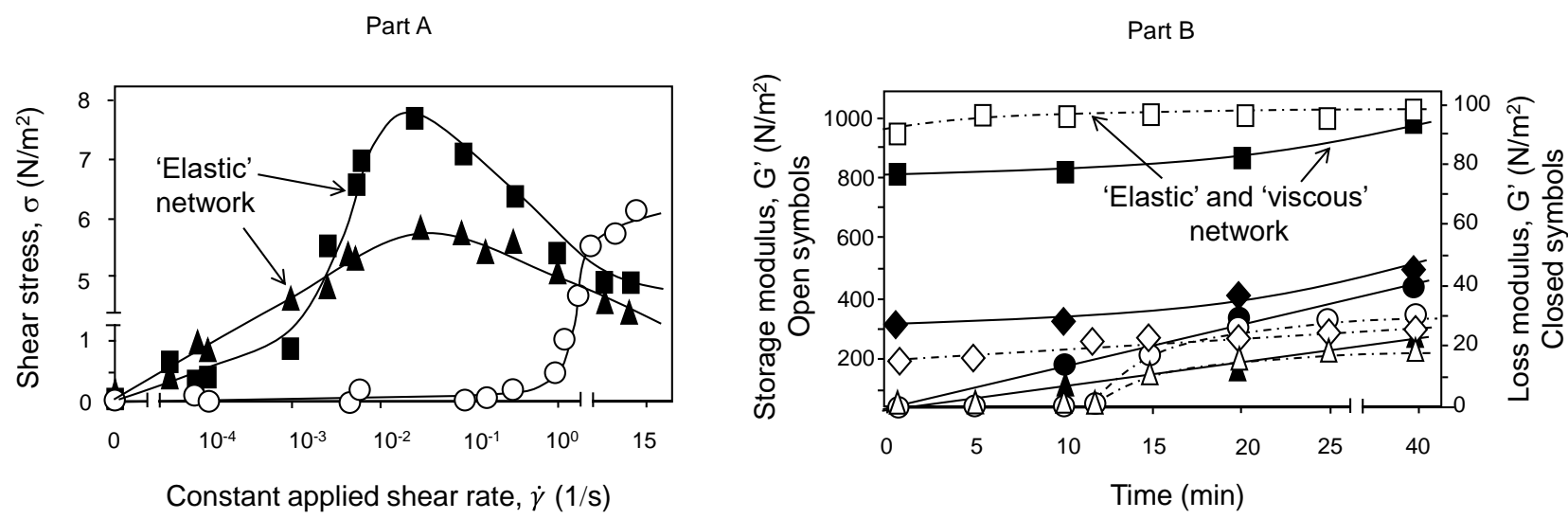

Fig. (5). The mechanical properties of oil-water $(\mathrm{O} / \mathrm{W})$ surfaces/macroscopic interfaces based on dilute dispersions of proteins and simple surfactant, Tween 20 in various combinations using a sharp-edged bicone measuring head located in the plane of the interface. The protein samples featured are, $22 \mu \mathrm{M}$ BSA ( $\mathbf{\square}), 26 \mu \mathrm{M}$ ovotransferrin $(\mathrm{CON})(\mathbf{\Delta})$ and $22 \mu \mathrm{M}$ BSA in the presence of $19.8 \mu \mathrm{M}$ Tween 20 (molar ratio for T20: BSA, $R=0.9)(O)$ ). Stressstrain data at $\mathrm{O} / \mathrm{W}$ interfaces are shown in part $\mathrm{A}$ and the results of oscillatory viscometry at $\mathrm{A} / \mathrm{W}$ interfaces are given in part B as a function of equilibration time. In Part B 'elasticity' is represented by open symbols and viscosity is represented by closed symbols), the same material shapes apply in figure Part A and B. The only modification to the samples present in Part B compared with Part A is the inclusion of $42 \mu \mathrm{M} \beta \mathrm{CAS}(\diamond, \diamond)$ in the former. All solutions are made in $0.05 \mathrm{M}$ phosphate buffer, $\mathrm{pH} 7.0$ unless otherwise stated. 

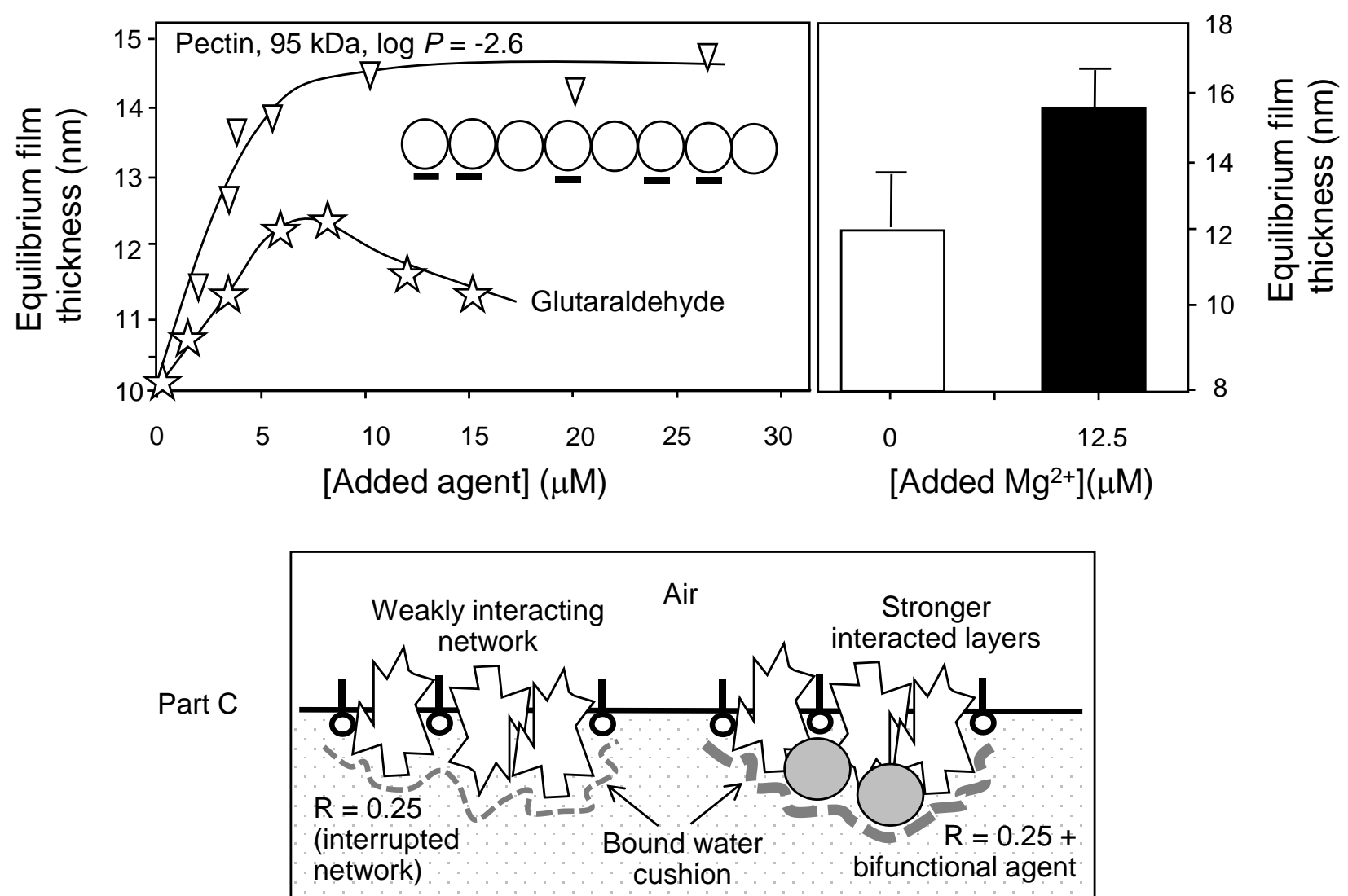

Fig. (6). The air-water (A/W) foam film (thin liquid film; TLF) values based on dilute dispersions of $50 \mu \mathrm{M} \beta$ lactoglobulin ( $\beta \mathrm{LG})$ protein and simple surfactant, Tween 20 (T20) in a combination of T20: $\beta \mathrm{LG}$ at molar ratio, $R$ $=0.25$ using an interferometric method with a monochromatic He-Ne laser light source in the presence of glutaraldehyde cross-linker ( 3 ) and negatively-charged pectin (Part A) and divalent magnesium ions (Part B). Phenomenological representation of the structures, particularly a bound water-cushion that serves to thicken and stabilise foam films is shown (Part C). All solutions are made in $0.05 \mathrm{M}$ phosphate buffer, $\mathrm{pH} 7.0$ unless otherwise stated.

ducing aggregate particles, which render the foam film unstable [27].

Insertion of divalent magnesium ions Fig. 6 (Part B), in a similar manner to the aluminum reported previously [27], also results in thicker foam films. This is assumed to occur because of bridging between ionized acidic amino acids within the protein and cations. Just as in the case of using other bifunctional agents, concentrations of crosslinker that are too high simply cause mass aggregation (illustrated by the fall-off in foam film thickness for the glutaraldehyde data in Fig. 6 (Part A)) and this tends to destabilize the interfaces of foam films within foams and inter-droplet liquid films in emulsions [23, 28]. This has numerous applications in terms of the minerals, such as potassium aluminum sulfate (alum) used in vac- cines, along with milk and egg proteins (see Table 1) and calcium or phosphate ions. The schematic representation of interfacial composition and structuring is presented in Fig. 6 (Part C). The Figure in cartoon form shows what happens to interfacial adsorbed protein, in-part disrupted in its continuity, by the inclusion of a competing small molecule surfactant, upon inclusion of a bifunctional agent [27]. Unification of protein-rich portions of the adsorbed layer allows "bridging around the impurity (surfactant)" and a partial or near-full reestablishment of protein network cohesion, waterbinding and prominence at the interface. The larger bound water cushion seen in the system with incorporated bifunctional agent, means thicker foam (and emulsion) films and greater short-term stability against coalescence and disproportiona- 
tion $[28,30,32,37]$. The consolidation of the protein part of the adsorbed layer or polymer, in the case of polymer incorporation, allows mixtures of large and small molecules to co-exist, where these compounds would be normally mutually incompatible.

\subsection{Probing the Adsorbed Layer}

In a foam film constituted from a mixture of $\beta$ lactoglobulin and Tween 20, at a molar ratio, $R=$ 0.3 , where disruption of the protein-protein interaction occurs but is not comprehensive it is possible to chart the freedom allowed to a trace incorporated fluorescent probe surfactant with a $\mathrm{C}_{18}$ aliphatic tail (ODAF) using a technique known as, fluorescence recovery after photo-bleaching (FRAP) $[2,27,41]$. In the presence of strong and comprehensive protein-protein interactions and crosslinking, "compartmentalization" and binding or restricted presence (estimated by pre-bleach fluorescence count) shows a restriction to movement of the probe molecule. The technique stands up well to direct labelling of the protein which changes the protein chemistry and surface activity [27].
The first part of the Figure (Fig. 7, Part A) shows what happens to the freedom of movement of the probe molecule, on inclusion of a compound isohumulone (which represents a sound model of phloroglucinol compounds, such as the antispasmodic pharmaceutical, flopropione). The reduced mobility of the probe, points solidly to the establishment of enhanced protein-protein interactions [27, 41] and the formation of a more extensive, robust and elastic adsorbed layer on establishment of crosslinks. Isohumulone (IH) also represents a good model of a drug compound having a $\log P$ of 5.3 and thus, clearly being poorly watersoluble and hydrophobic in nature, like a whole swathe of pharmaceutical actives. The molecule is represented in cartoon, simplified form in the Fig. (Part A) and is based on an apolar core, aliphatic side chains and three minor hydrophilic groups that are thought to be responsible for the crosslinks $[17,21,25,42,43,51]$ and alter inter-species interactions and subsequent mobility.

The Figure also shows in Part B, the surface tension combined with dilatational elasticity and the dilatational viscosity of an $\mathrm{A} / \mathrm{W}$ macroscopic interface, and the effect of a fixed concentration of

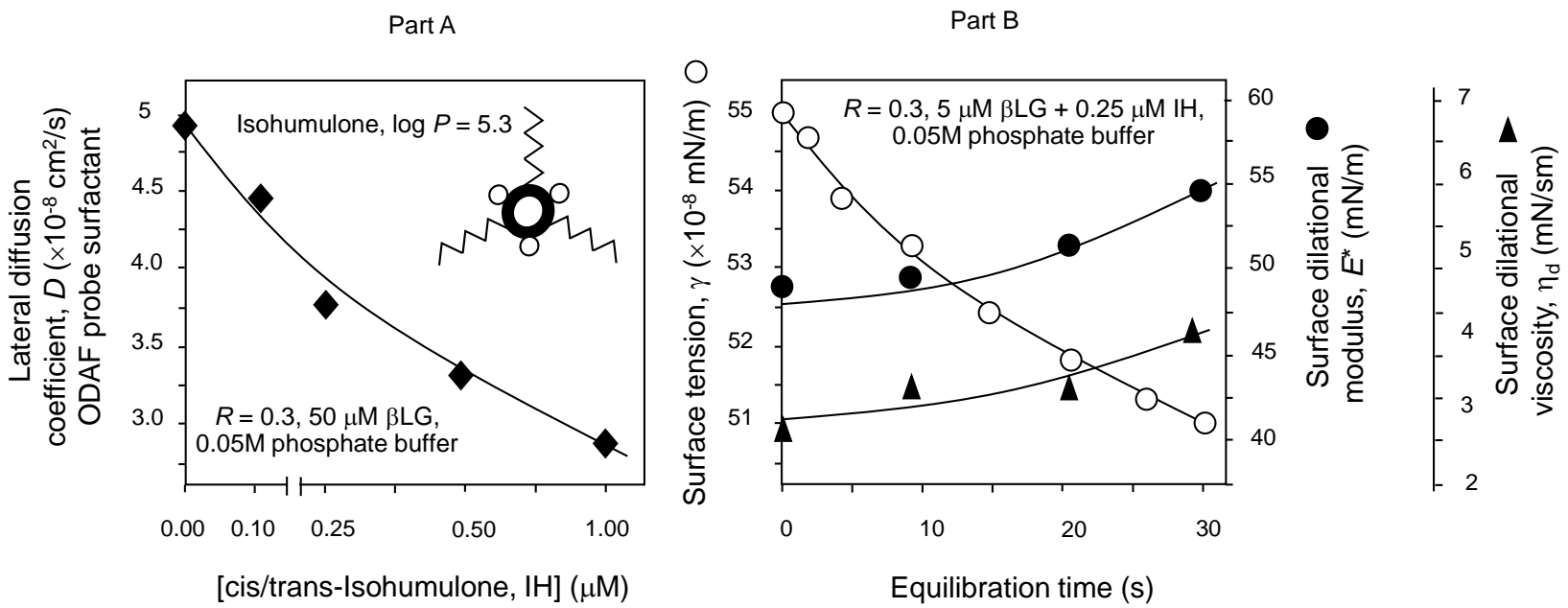

Fig. (7). The molecular diffusion of a fluorescent interfacial probe molecule, ODAF in thin liquid films (TLFs) based on dilute dispersions of $50 \mu \mathrm{M} \beta$-lactoglobulin ( $\beta \mathrm{LG}$ ) protein and simple surfactant, Tween 20 (T20) in a combination of T20: $\beta \mathrm{LG}$ at molar ratio, $R=0.3$ in the presence and absence of a hydrophobic drug analogue (octanol-water partition coefficient, $\log P=5.3$ ), based on an aryl ring or similar apolar structure (heavy bordered circle), aliphatic side chains (zig-zag) and hydrophilic functional groups (small circle) see schematic in Part A. The hydrophobe is, isohumulone, isolated from hops. Molecular mobility and freedom of movement is evaluated using a FRAP photo-physics method of adsorbed layer interrogation. The surface tension of the $R=0.3$ sample, and its surface elasticity and surface viscosity assessed using surface dilation of a macroscopic A/W interface is shown with respect to surface-aging and equilibration time (Part B). The terms dilational and dilatational are interchangeable. All solutions are made in $0.05 \mathrm{M}$ phosphate buffer, $\mathrm{pH} 7.0$ unless otherwise stated. 
Tween 20 and IH. Very noticeably, addition of the crosslinker causes the surface tension to decrease and better residence of protein at the interface, and bearing in mind the system contains competing surfactant that disrupts the protein-protein interactions [3, 40, 42]. The adsorption of a mixture of protein, Tween, protein-Tween complex, protein$\mathrm{IH}$ and $\mathrm{IH}$ is manifested as an increase in both surface elasticity and viscosity [26, 43, 49]. This aging process is revealed even over the short timeframe of the measurement but noted to take place to a much greater degree on longer timed study described by Gao et al. [30].

\subsubsection{FRAP, SEM and AFM}

The photo-physics technique FRAP has some profound supportive properties of use, many over traditional rheological measurements (constant or damped surface shear, surface oscillation or surface dilation) that by virtue of distorting the adsorbed layer and that take place as part of the measurement itself $[34,52]$. The properties change and material form can change too, as part of the measurement, such as promoting viscosity changes. These are assumed to be associated with interfacial aggregation, driven by the mechanical action of portions of the adsorbed surface layer. FRAP can be used, and has been used in 'rheological' context [41] because it measures the 'freedom' permitted to the smaller mobile species in the plane of the interface (Fig. 8, Part A). In this way, slowing of interfacial mobility for the fluorophore ODAF, means a greater disruption of interfacial protein-protein interactions and a change in composition, which $\mathrm{O} / \mathrm{W}$ surface shear (Fig. 5, Part A), A/W surface tension measurements (Fig. 3, Part A) and A/W foam composition measurements (Fig. 4, Part A), illustrate is principally dictated by competing low molecular weight (Tween $20)$ or simple polymeric $(\mathrm{MeC})$ surfactant $[3,11$, $19,27,41]$ and replacement of one species preferentially by another.

Molecular mobility and entrapment of the fluorescent probe surfactant, ODAF is shown in Fig. 8 (Part A). The broken lines describe the mobility of the probe in $0.05 \mathrm{M}$ imidazole buffered medium (different to all other data) in the presence of trivalent aluminum ions, associated with a partly disrupted interfacial adsorbed layer (on increasing increments of low molecular weight surfactant). The ability of ions to crosslink adsorbed interfacial protein has been described previously [27, 28, 30]. The plot shows the presence of $5 \mu \mathrm{M}$ aluminum ions changes the onset of increased interfacial mobility $(D)$ from molar ratio $R=0.5$ to $R=0.95$. This is a significant elucidation of the role that simple ions can play in pharmaceutical and vaccine formulations such as those containing the aluminum salt, alum. Similarly, cations and selenides were reacted to stabilize the surface of nanoemulsions in the work of Piętka-Ottlik et al. [24]. In a roughly equivalent manner, the plot also shows the effect of inclusion of $5 \mu \mathrm{M}(+)$-catechin, a hydrophilic polyphenol and close mimic of numerous quinolone antibiotics, such as ciprofloxacin and flavone derivatives, such as apigenin [4, $38,44,47,52]$ have the ability to act as bifunctional agents. The polyphenol changes the onset of 'loosening' of the interfacial structure (measured as probe molecule increased mobility) from 0.25 $\mu \mathrm{M}$ added Tween 20 in $\beta \mathrm{LG}$ structured foam films to $0.3 \mu \mathrm{M}$. Notably in the Figure the error bars are of a size, at the largest, approximately equal to the size of the symbols and therefore, indicate this ' $2 \mathrm{D}$ gelation' and consolidation of the adsorbed interfacial protein to be a real finding, as previously reported for functionalized polysaccharides in emulsions $[19,32]$.

Interfacial rheology involving hydrophilic polyphenols ( $\log P \sim 1)$, such as (+)-catechin or structurally similar pharmaceuticals, can have a drastic effect on the protein-protein interactions at the surface of a dilute $5 \mu \mathrm{M} \beta \mathrm{LG}$ solution with included Tween 20 , at $R=0.25$. Both the surface 'elasticity' (dilatational or dilational modulus) and surface 'viscosity' (dilational viscosity) increase on addition of submicromolar concentrations of catechin, with moderate concentrations of polyphenol on the plot producing enhanced elasticity, yet only marginally increased surface viscosity. These findings are entirely consistent with enhanced protein-protein interactions initiated by inclusion of the polyphenol [14, 47, 48]. Modelling and explanation of the disruption and re-unification of adsorbed interfacial protein and polymers has been widely reported [3, 27, 28, $36,41,42]$ across a range of spectroscopic and surface rheological studies.

Surface shear rheological data is presented in Fig. 9. The plots indicate that the two-dimensional 'gelation' of adsorbed protein at an $\mathrm{A} / \mathrm{W}$ interface form pure protein (BSA), mixtures of protein and Tween 20, the mixture in the presence of a cereal 
Part A

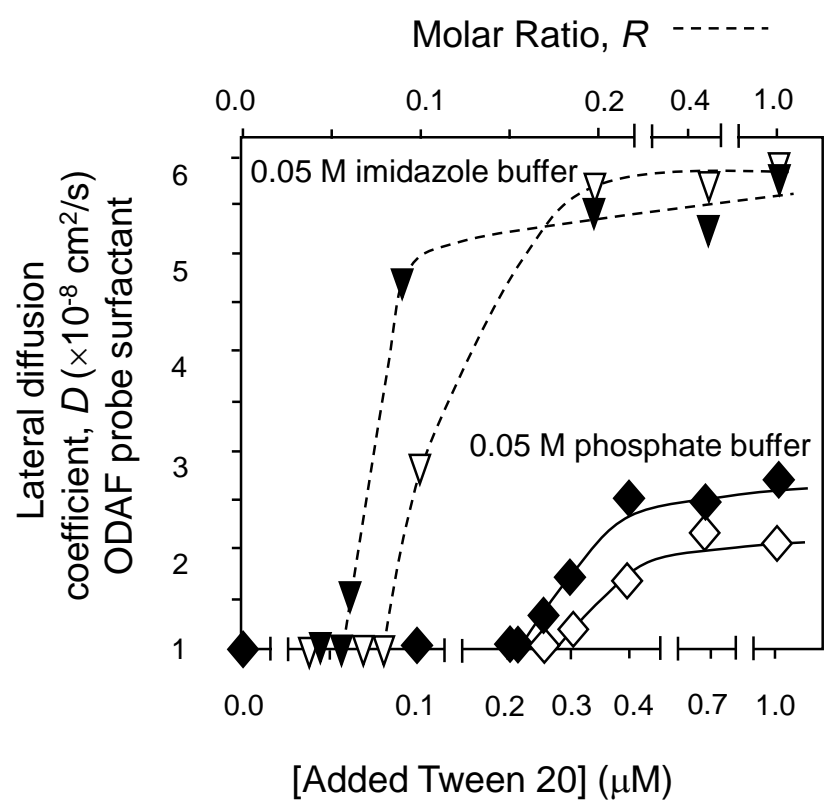

Part B

$R=0.25,5 \mu \mathrm{M} \beta \mathrm{LG}$, $0.05 \mathrm{M}$ phosphate buffer

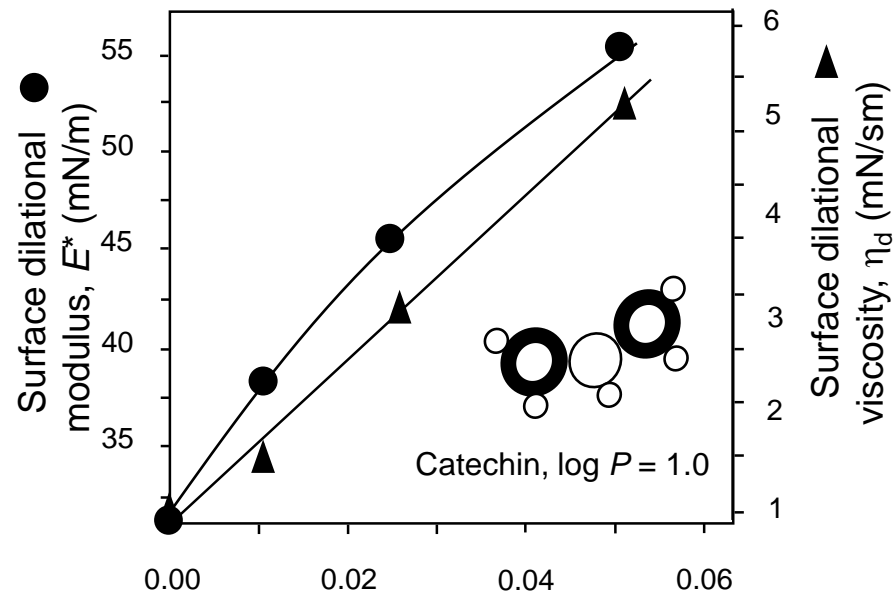

[Added (+)-Catechin] $(\mu \mathrm{M})$

Fig. (8). Part A: The molecular diffusion of an interfacial probe molecule, ODAF in thin liquid films (TLFs) based on dilute dispersions of $50 \mu \mathrm{M} \beta$-lactoglobulin ( $\beta \mathrm{LG}$ ) protein and simple surfactant, Tween 20 (T20) in a combination of T20: $\beta \mathrm{LG}$ at molar ratio, $R=0.25$ in the presence $(\nabla)$ and absence $(\boldsymbol{\nabla})$ of $5 \mu \mathrm{M}$ trivalent aluminum ions as a model of the interaction between vaccine proteins and the formulation aid "alum" (triangles) - notably in $0.05 \mathrm{M}$ imidazole buffer at $\mathrm{pH}$ 7.0 , and dilute dispersions of $50 \mu \mathrm{M} \beta$-lactoglobulin ( $\beta \mathrm{LG}$ ) protein and simple surfactant, Tween 20 (T20) in a combination in the presence $(\diamond)$ and absence $(\diamond)$ of $5 \mu \mathrm{M}$ hydrophilic polyphenol (+)-catechin, a model for numerous drug molecules ( $\log P=1.02$ ), based on an aryl ring (heavy bordered circle) and hydrophilic functional groups (small circle) (see schematic in Part B). The effect of differing inclusion of (+)-catechin for a $5 \mu \mathrm{M} \beta$-lactoglobulin ( $\beta \mathrm{LG}$ ) protein solution of Tween 20 inclusion at $R=0.25$, and its surface elasticity and surface viscosity assessed using surface dilation (Part B). All solutions are made in $0.05 \mathrm{M}$ phosphate buffer, $\mathrm{pH} 7.0$ unless otherwise stated.

pentosan (with molecule shown schematically in the Figure) and the mixture in the presence of catechin. The natural rye pentosan is chemically derivatized, with periodic attachment of the ferulic acid polyphenol $(\log P=1.6)$ to the polymer backbone [14, 36, 48]. Pure protein and peptide or hydrophobic and lipophilic protein 'aggregate', has a considerable surface shear viscosity and elasticity [26, 30, 43], consistent with 'firm' protein-protein interactions and may be present as a mix of monolayer or monolayer and entrained interfacial aggregates. Making A/W macroscopic interfaces from mixtures of protein and small molecule surfactant, reduces both the viscosity and elasticity of the adsorbed interfacial layer in a manner entirely consistent with the phenomenon of competitive displacement and competitive adsorption [3, 41]. Arabinoxylan bears considerable similarity $[14,15]$, in terms of molecular form, to a range API's of the macrolide class of antibiotics such as, erythromycin and azithromycin, and is not dissimilar in a crude sense, to $\beta$-lactam molecules, such as penicillin and benzylpenicillin [39], although it is somewhat larger in size (macrolides and $\beta$-lactams are approximately $0.1 \%$ of the size of the pentosan).

Equilibration of solutions containing a much higher proportion of Tween 20, compared to protein in Tween 20/BSA solutions, $R=1.5$ with a concentration of $3 \mu \mathrm{M}$ highly water-dispersible arabinoxylan $(\log P=-3.2)$ incorporated are presented in the inset in Fig. 9. With time, the elasticity of the surface adsorbed layer increases, while the viscosity is decreased. Again, this is entirely consistent with the forging of mediated protein-protein interactions in the plane of the adsorbed layer and supports findings from interferometric measurements of foam film thickness, surface shear rheology and FRAP studies [3, 27, 41, 42], which chart the surface chemical composition and its effect on rigidity. Oth- 


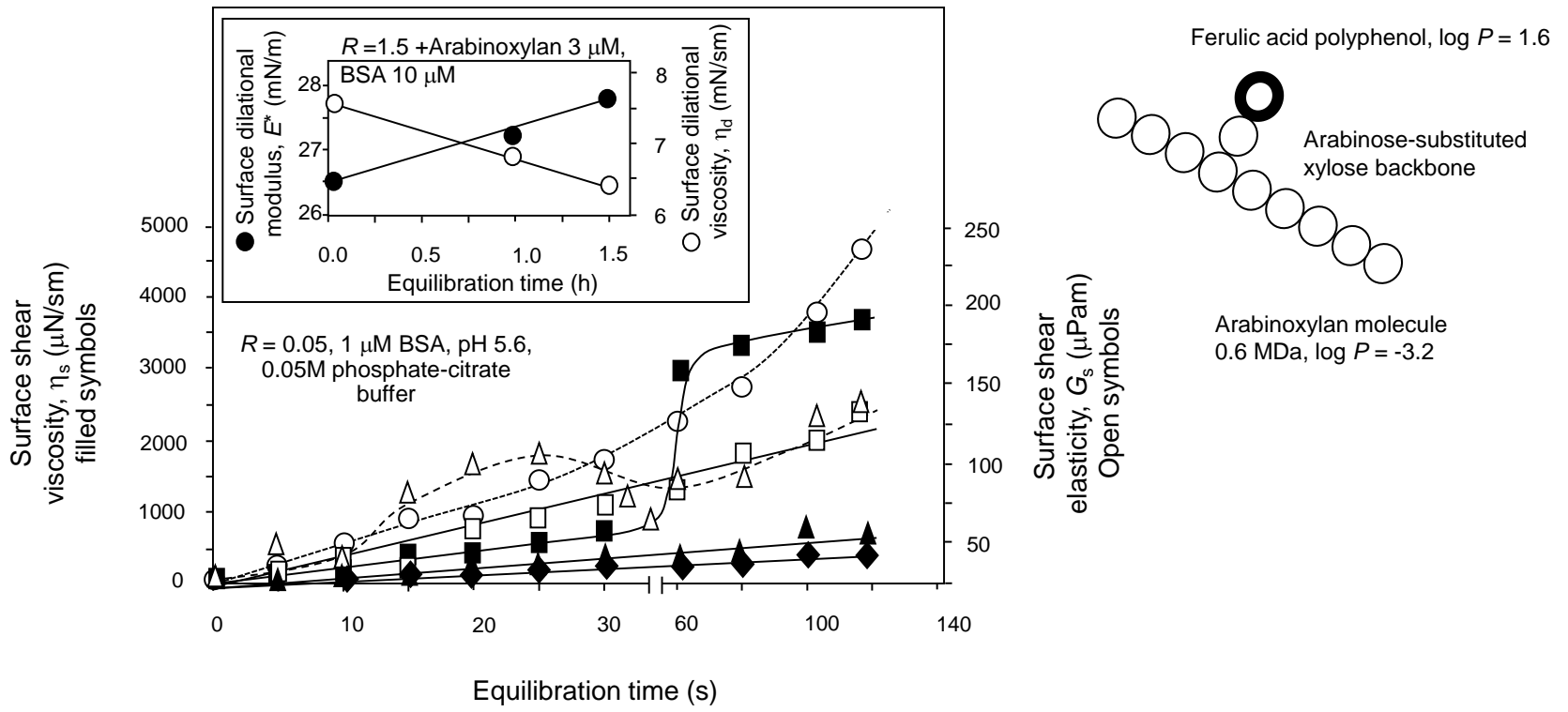

Fig. (9). The mechanical properties of air-water (A/W) surfaces/macroscopic interfaces based on dilute dispersions of proteins and simple surfactant, Tween 20 in various combinations using a sharp-edged measuring head located in the plane of the interface (undertaken at the Max-Planck Institute in Berlin on a customised device). The protein samples featured are, $1 \mu \mathrm{M}$ BSA (O, elasticity), $1 \mu \mathrm{M}$ BSA + T20, $R=0.05$ ( $\boldsymbol{\square}$, viscosity) and ( $\square$, elasticity). The $R=0.05$ sample with $1 \mathrm{nM}$ hydrophilic polymer with ferulate-hydrophobic side chain (shown in the schematic), arabinoxylan $(\mathrm{AXN})$ polymer added in terms of its elasticity $(\Delta)$ and viscosity $(\boldsymbol{\Delta})$ is also shown alongside the viscosity of $0.1 \mu \mathrm{M}(+)$-catechin $(\diamond)$. The inset shows the improved mechanical effect of arabinoxylan inclusion on much more fractured $R=1.5$ adsorbed layer surfaces in the presence of $3 \mu \mathrm{M}$ arabinoxylan polymer as a function of standing time.

er techniques, such as atomic force microscopy (AFM) and scanning electron microscopy (SEM) can also be used alongside more traditional forms of surface structure evaluation by applying as little as piconewton forces $[2,53]$.

Microscope-based examination of the materials adsorbed on the surface of $200 \mathrm{~nm}$ polystyrene latex nanobeads, can be used to deduce the composition and mechanical properties of adsorbed material. Techniques such as, AFM and SEM can be used to describe the architecture of polymers builton the bare nanobeads (Fig. 10, Part A). AFM is perhaps more useful because the tapping mode of measurement (cantilever resonance $\sim 295 \mathrm{kHz}$ ) can be used to deduce the phase lag in the vertical plane between input and output signal and therefore, deduce a change to any tip oscillation through 'damping actions' from material being either absent (hard contact) or present (soft surface) on the bare particle surface (Part B). Block copolymer of the form illustrated in a highly generalized cartoon form in Fig. 10 (Part C), such as Pluronics (poloxamers) and Tetronics (poloxamines) are highly surface-active and adhere to hy- drophobic nanoparticles of substrates, such as polystyrene latexes $[9,19,20,44,46,50]$ to form a surface cushioning. The form of surface-active polymer adsorbed on surface, which if then dried to equilibrium humidity, preserves the 'solution form' of the polymer and consequently, allows the near-dry samples to be prepared for a 'dry-type' of examination.

The damping quantifiable by phase lag measurements can be used to chart the form of the surface-active block copolymer adsorbed on the bead (Fig. 10, Part C). Part C shows the phase lag for 3 different surface polymer compositions; line 1 represents the form seen for a bare latex nanobead, line 2 for the same beads but now covered with polymer from a solution containing $55 \mu \mathrm{g} / \mathrm{ml}$ poloxamine and line 3 for the same beads with polymer from a solution containing $300 \mu \mathrm{g} / \mathrm{ml}$ poloxamine. The significant part of the plot really lies in the data accrued from the middle or top of the particle, before the shape of the spherical bead curves away from the measurement tip ( $h=0$ to 60 $\mathrm{nm})$. Tangential contact with the bead with the AFM tip, also contains rheological information but 
is not as clear to decipher [2]. Line 1 (image C) represents a hard, high phase lag surface of a bare bead $\left(\beta \sim 37^{\circ}\right)$ and as increasing amounts of polymer are adsorbed, line 2 and line 3 (image D), respectively, on the particle surface, in equilibrium with contents of the dispersion solvent the phase lag is altered from that of the bare particle. Hypothesizing the practical meaning of lines 2 and 3 , this suggests the polymer molecule is adsorbed in flat pancake-like surface $\left(\delta \sim 12^{\circ}\right)$ hugging and extended $\left(\delta \sim 8^{\circ}\right)$ pendant (brush) forms, the latter being the least damping, which does not modulate AFM tip oscillation as much as the bare surface. These findings are confirmed by assays have been used to directly measure the loading of polymer on a latex material [54].

\subsection{Dissolution of Molecular Interactions}

The weight of evidence now pointing to the disruption of surface adsorbed polymer by small molecule surfactants is now considerable and extensive, covering a period of more than 20 years $[3,8$, $11,41,50]$. Disruption of networks of principal component and the fabrication of channels, in which therapeutics is loaded, is now a real possibility. Combinations of studies involving surface tension estimation at $\mathrm{A} / \mathrm{W}$ and $\mathrm{O} / \mathrm{W}$ interfaces, surface shear and dilatational rheology, interfero-
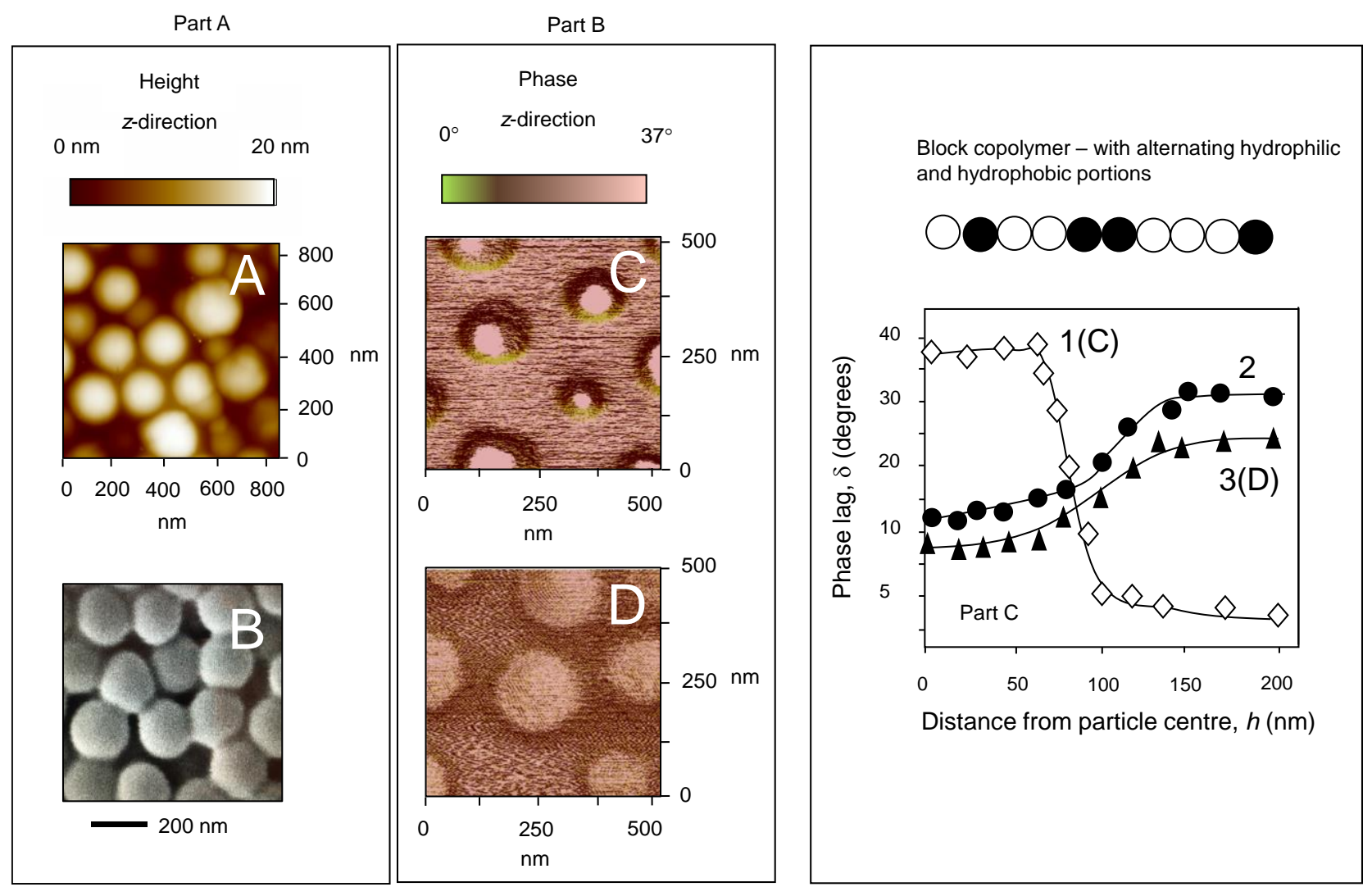

Fig. (10). Part A: top to bottom, atomic force microscopy (AFM) in tapping mode of polystyrene latex nanobeads dried to 55\% relative humidity from solutions. Image A shows an AFM image of beads measured in terms of vertical height, in the presence of Pluronic F108 adsorbed polymer and image B shows the same latex beads but captured using scanning electron microscopy (SEM), at magnification x15000, these serve as control images. Part B from top to bottom, shows the phase lag data captured by tapping mode data collection. Panel $\mathrm{C}$ shows bare latex beads of about $200 \mathrm{~nm}$ diameter; panel $\mathrm{D}$ shows the same beads but covered with a loading of a solution containing $300 \mu \mathrm{g} / \mathrm{ml}$ poloxamine 908 hydrophilic block copolymer. The polymer is frequently used in drug delivery applications. Part $\mathrm{C}$ shows direct nano-rheology data (phase lag) in traversing the surface of the bead from the centre to its margins. In the plot, line $1(\diamond)$, refers to image $\mathrm{C}$, line $2(\bullet)$ to a surface loading achieved in a $55 \mu \mathrm{g} / \mathrm{ml}$ poloxamine 908 and line $3(\boldsymbol{\Delta})$ is from image D. High values in Part C represent no loss of signal and low values represent a damping of the movement of the probe tip synonymous with a soft palisade layer of adsorbed material. All solutions are made in $0.05 \mathrm{M}$ phosphate buffer, $\mathrm{pH} 7.0$ unless otherwise stated. 
metric measurements of foam film thickness, FRAP and AFM can now be used alongside complementary techniques, such as photon correlation spectroscopy (PCS; light scattering) to reveal insights into molecular interaction in the plane of the adsorbed layer material. Dissolution or partdissolution of the array of interactions between and within complex hetero-polymer molecule networks, such as adsorbed proteins and nucleotides is entirely feasible. Therefore, it is possible to engineer the properties of therapeutic foams and emulsions [5, 8, 11, 12, 16, 20, 22, 43, 45, 47, 55] by using complementary mixes of amphiphilic soft matter assemblies and solid suspensions to 'house' drug in the adsorbed layer, which adds another possibility for the notions of a controlled release of drug, particle loading with therapeutics, encapsulation $[10,16,39,45,46,47]$ of valuable species and also for housing quantum dots and plasmon generating nanoparticles for diagnostic medical uses described at length by Bazylińska et al. [56].

\section{BIFUNCTIONAL AGENTS AND THEIR ROLE IN INTERACTIONS WITHIN THE 'FRACTURED' ADSORBED LAYER}

A number of publications now consider the use of bifunctional agent, which if suitably chemically charged or functionalized, with respect to a substrate can be used to crosslink interfacial species and restore or optimize rheological properties [34]. The restoration can be based on hydrogen bonding or formal covalent bond formation, as possible with amino-carbonyl bond formation e.g. glutaraldehyde and the amine groups of lysine, in proteins or equivalent groups in polymeric surfactants (Figs. 6-8) and therapeutics. Restoration can be based, in part on complexation of competing surfactant by a polymer, such as a protein, thus effectively removing the disruption of the proteinprotein interactions [34, 43]. Ion-pair formation as a result of electrostatic interaction is also possible for polyions [45] and simple species (Fig. 6).

When bifunctional agents are also therapeutics, such as insulin or polyphenol analogues (flurbiprofen, ketoprofen, pine tannins [57]) and oxidized polyphenols/quinones (e.g. quinolone drugs, such as ciprofloxacin), then loading of the adsorbed stabilizing layer of foams and emulsions is made entirely possible. The loading is further aided if the therapeutic is surface-active, such as lidocaine, amitriptyline, methotrexate, insulin, nerve growth- stimulating polyphenols (e.g. apigenin [52]), OTC terpenoids (e.g. citral, levomenthol, limonene), psycho-active phenothiazines [10] (e.g. thorazine and stelazine) and phloroglucinol. Conveniently, many drug compounds contain unsaturated aromatic rings and aliphatic portions, and are often heterocyclic in character but with polar functional groups such as carboxylic acids groups, amines and hydroxyl groups that lend themselves to providing some degree of surface-activity [5, 17, 38 ] to the molecule. Partition coefficients, such as $\log P$ are usually a good indicator or surfaceactivity in simple molecules, with values of zero to one, usually indicating water solubility and values of three to six usually indicating poor water solubility or dispersibility. This is not always clear with proteins and peptide drugs, as they contain a mix of hydrophobic and hydrophilic amino acids and thus often have low $\log P$ values, yet act as very good polymeric surfactants $[28,33,34,40$, 41] as evidenced in foam formation because of possessing hydrophobic amino acid-enriched portions and domains.

\section{APPLICATION OF PRINCIPLES TO DRUG BASED MODELS}

Using simple models of interfacial structuring and composition, delineated by a number of diverse physical techniques it is possible to describe and characterize the surface of coarse dispersions used in therapeutic or functionalized systems described by Thomas et al. [58]. Solid dispersions of drug particles, therapeutic foams, such as those based on cortico-steroidal drugs used to treat local inflammation, irritable bowel syndrome, and eczema and a vast array of emulsions used to solubilize therapeutic species, such as OTC fat soluble vitamins, hugely apolar OTC antifungal drugs (miconazole and terbinafine) and hormones, such as estradiol and testosterone frequently use polymeric and simple emulsifiers. In fact, this blending of the two or more 'surfactants' has been used for some time in pharmacy to give a surface covering of droplets with a combined surface loading to maximize process stability $[9,12]$. The natural extension to this notion, is to use the drug (surfactant-like in character) to interact with the principle surface-active agent used to fabricate pharmaceuticals and vaccines. These simple emulsifiers and larger surfactants are very often polymeric in nature, such as alginate $[14,45]$ and used in thermosensitive applications, the hydroxypropyl version 
of methylcellulose [19] (hypromellose), acrylates (Tergitol, Carbopol) or amphiphiles based on poly(ethylene)-poly(propylene) oxides (Pluronics) are also used in pharmaceuticals [22, 43]. Such excipients are frequently pharmaceutical grade polymeric surfactants or the proteins, such as gelatin, egg albumin or milk proteins in vaccines.

\subsection{Interfacial Complexation of Drugs}

A background understanding (Fig. 1 and Figs. 39) of interfacial coverage by surface active polymers can be used to garner an understanding of the behavior of polymers [48] and wetting or surfacestabilizing agents in therapeutic products. In addition, studies involving bifunctional agents, such as alginates $[24,27,45]$ are helpful; in that they describe the manner in which, substances can be incorporated into the adsorbed layer of a foam film or emulsion droplet, whilst retaining a therapeutic bioavailability. Medicinal products are complex commodities with 'actives' and excipient components. Vaccines and peptide hormone-based pharmaceuticals are yet more complex, as maintaining the molecular integrity of the proteins, polysaccharides, surfactants and lipids (most of the components listed in Table 2, with the exception of ODAF) is absolutely essential to their biological functioning [9]. Such complex component mixtures with varying interfacial compositions and architectures [19, 20, $22,55]$ of rafts, suprastructure and channels are not only pre-requisites of therapeutic efficacy but also of suitability, compatibility and quality $[1,2,9,11$, $17,35]$ with features that aid active sequestering and encapsulation. Architectures can be made to be 'trigger' release or activation of a drug as a result of local conditions, such as by chemical modification, thus possessing sensitivity or responsiveness to temperature $[22,45,49]$ or $\mathrm{pH}$ changes as seen with polysiloxanes [21].

Fig. 11 is complex and comprises of three main parts. Part A shows the A/W surface tension versus concentration for two well-used therapeutics, lidocaine and amitriptyline. Both drugs are hydrophobic in nature (represented phenomenologically in the cartoon). As such, they both demonstrate considerable surface-activity on their own. Both surface tension plots show the surface tension line for the Pluronic surfactant F68 (Flocor), used ubiquitously in pharmaceutical and biomedical dispersions. Interestingly, as observed in the previous studies with lecithin and protein [41], cross- over of the line for a mix of a set concentration and increasing addition of drug and those of the pure drug and pure polymer hint at the formation of a surface-active 'complex' [4, 38] (or at least an 'associated' entity), which is able to sometimes lower the surface tension better fashion than the polymer it contains (top plot) or less well than either the pure polymer or pure drug (bottom plot) at some critical concentration.

\subsection{Inclusion of Drug Molecules in Interfacial Architectures}

Part B of Fig. 11 shows surface tension at the top, and macroscopic interface surface shear viscosity at the bottom. Both plots are for the steroid drug, betamethasone valerate, Pluronic F108, steroid, steroid and Pluronic (with fixed Pluronic concentration) and lysophosphatidylcholine (LPC), used for comparison. The top plot shows the A/W surface tension of a mixture of betamethasone and fixed Pluronic concentration to be lower, at equivalent concentrations to that of drug alone, Flocor alone or LPC alone across an increasing concentration of steroid. This means that the 'molecular hybrid' of betamethasone and Pluronic is more surface-active than any of the individual components. Notably, in the surface shear data, the interfacial viscosity of the mixed system is higher than that of either the pure drug or pure polymer and much greater than that of LPC. A significant change in interfacial structuring $[3,33,49,52]$ and the magnitude of lateral interactions between adjacent adsorbed molecular species of the polymer adsorbed layer can be inferred from this rheological data involving inclusion of the drug. Fig. 11 (Part C) shows the surface tension at the top and solid-water (S/W) particle diameter measurements using PCS light scattering at the bottom. The particle sizes represented are those accounting for the hydrodynamic diameter of adsorbed layer built on to a spherical silica core. Silica is frequently used in pharmacy to bind antibiotics by crosslinking or association, as reported for propylacrylamide [58], also prior to formulation of a coarse dispersion of drug particles with sterile water, and to aid uniform dispersal throughout the medium [1, 29]. Again, as with the betamethasone, the poloxamerinsulin combination is shown to be highly surfaceactive and distinct from the curve for either pure poloxamer or pure drug. The indication is that a molecular complex formed between the insulin in terms of its hydrophilic (e.g. serine) and hydro- 
phobic (e.g. tyrosine) amino acids and the hydrophilic and hydrophobic portions of the poloxamer. The lower element in Part $\mathrm{C}$ shows how the average particle diameter is consistently (error bars are of the size of the symbols) higher, when netnegatively charged insulin is combined with cationic chitosan [9] and hydrophilic-hydrophobic Pluronic, than particle coverage with any mixture containing only one additional ingredient in addition to the silica. This points at the formation of possible multilayers of protein and other polymers and the formation of a complex architecture revealed by the increase in diameter [41].

\subsection{Drug Delivery Nanoparticles and Lipo- somes}

Fig. 12 is based on describing the encapsulation of cytotoxic anticancer drugs in the adsorbed layers of surfaces in A/W dispersions, measured using surface tension and the S/W interfaces of liposomes formed from the membrane phospholipid di-palmitoylphosphatidylcholine (DPPC) and cholesterol, a membrane intercalation aid and leaflet stiffener $[2,38]$, measured using PCS light scattering. The complex [11] formed between the drug methotrexate and Pluronic F68 shows a distinct difference to that of either pure drug or pure poly-
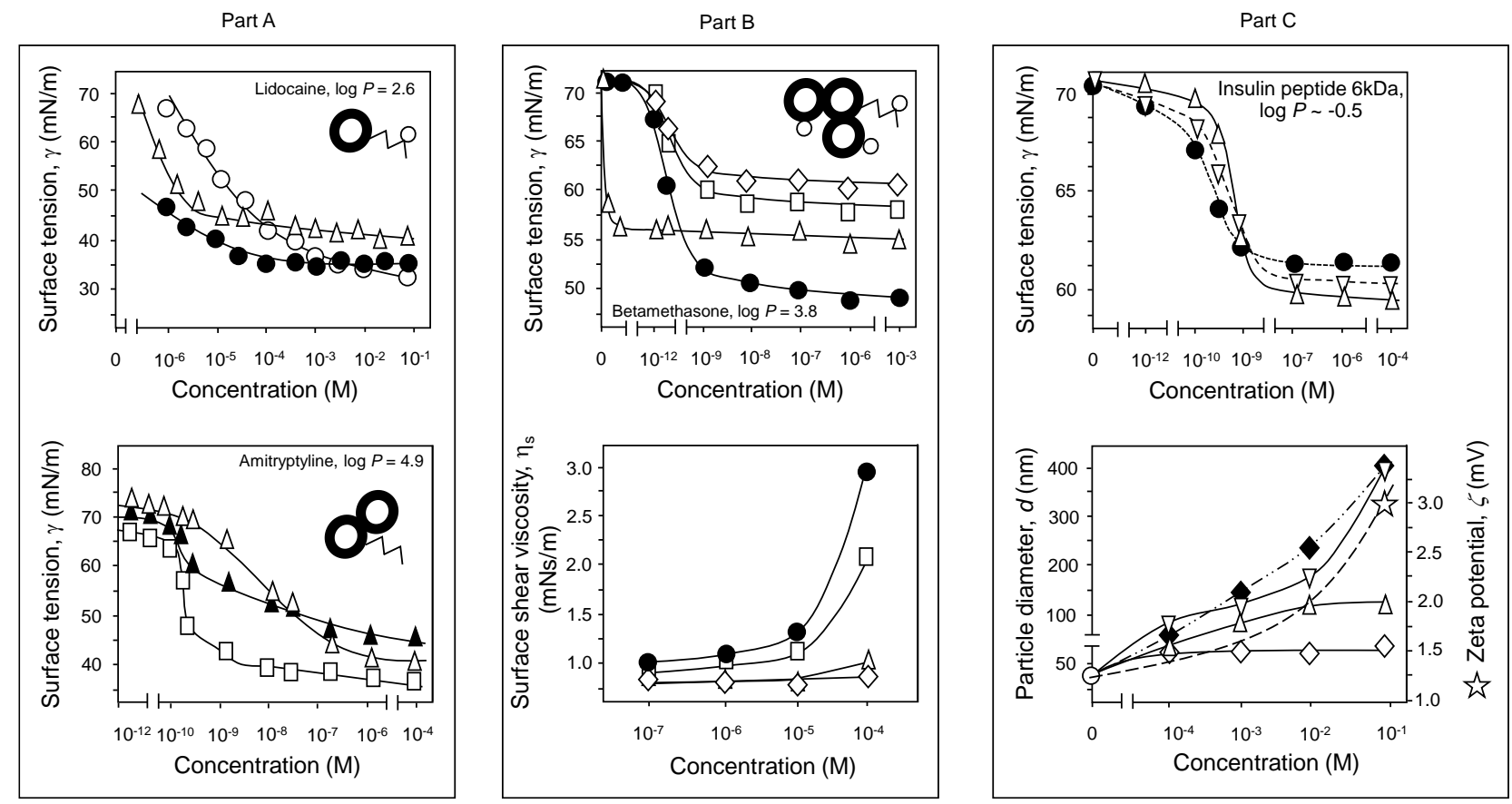

Fig. (11). Part A: shows the surface tension crossover for a mixed sample of increasing moderately hydrophobic anaesthetic drug lidocaine and with fixed $10^{-3} \mathrm{M}$ polymer Pluronic F68 $(\mathbf{O})$ and F68 alone $(\Delta)$ in surface tension in the top plot for moderately hydrophobic lidocaine $(\mathrm{O})$ having a $\log P$ of 2.6. In the bottom surface tension plot for the data for hydrophobic amitriptyline alone $(\square)$ and in the presence of $10^{-10} \mathrm{M}$ Pluronic F68 $(\Delta)$ is also represented alongside F68 alone $(\boldsymbol{\Delta})$. Part B: top panel shows surface tension, with $\log P=3.8$ hydrophobic corticosteroidal drug betamethasone-17-valerate $(\diamond)$, polymer Pluronic F108 $(\square), 10^{-10}$ M Pluronic F108 with variable betamethasone-17-valerate $(\Theta)$ and 2:1 palmitoyl-stearoyl L- $\alpha$-lysophosphatidylcholine, LPC $(\Delta)$. The bottom panel of Part B shows the surface shear viscosity for the cortico-steroidal drug betamethasone-17-valerate $(\diamond)$, polymer Pluronic F108 ( $\square$ ), $10^{-6} \mathrm{M}$ Pluronic F108 with variable betamethasone phosphate (O) and L- $\alpha$ lysophosphatidylcholine, LPC $(\Delta)$, respectively. Part C: top panel shows the polar, water dispersible $(\log P \sim-0.5)$ therapeutic protein insulin $(\Delta)$, surface active polymer Pluronic F108 $(\nabla)$ and a mix of $10^{-10}$ M Pluronic F108 with variable insulin $(\mathbf{)})$. The bottom panel of Part $\mathrm{C}$ shows the increase in hydrodynamic diameter of near neutral charged spherical silica nanoparticles as determined by light scattering on inclusion of $5 \mathrm{kDa}$ moderate molecular weight chitosan $(\diamond)$, surface active hydrophilic polymer, Pluronic F108 $(\Delta), 5.8 \mathrm{kDa}$ insulin $(\nabla)$, and a mixture involving $10^{-3} \mathrm{M}$ Pluronic F108, $10^{-3} \mathrm{M}$ chitosan and increasing concentrations of insulin $(\diamond)$. The zeta-potential or surface charge of the mixture is also indicated. All solutions are made in $0.05 \mathrm{M}$ phosphate buffer, $\mathrm{pH} 7.0$ unless otherwise stated. 
meric surfactant and indicates the formation of a complex (Part A). It is possible that there is a covalent linkage between drug and polymer, since the drug has several amine groups within its structure and the ether or free hydroxyl group of the polymer may allow a formal molecular interaction. This synergistic relationship is also shown to be the case when liposome hydrodynamic diameter was measured and this is thought to be associated with the formation of multiple consecutive layers in a process known as layer-by-layer fabrication $[9,29]$ of lecithin, poloxamer and charged drug.

Fig. 12 (Part B) shows the surface tension data for doxorubicin and the hydrodynamic diameter of liposomes again based on DPPC and cholesterol in the presence and absence of drug. The surface tension data points clearly at the formation of a drug Pluronic F108 complex because of the cross-over in the surface tension plot whilst also showing a difference between fixed drug quantity and increasing polymer and fixed polymer and increasing drug content, varying the drug content give a line, which is dissimilar from the pure drug but also distinct from the line for increase polymer. The size data measured at the vesicle S/W interface shows the difference in DPPC vesicle size on addition of both polymer and doxorubicin over and beyond that of the doxorubicin on its own. This
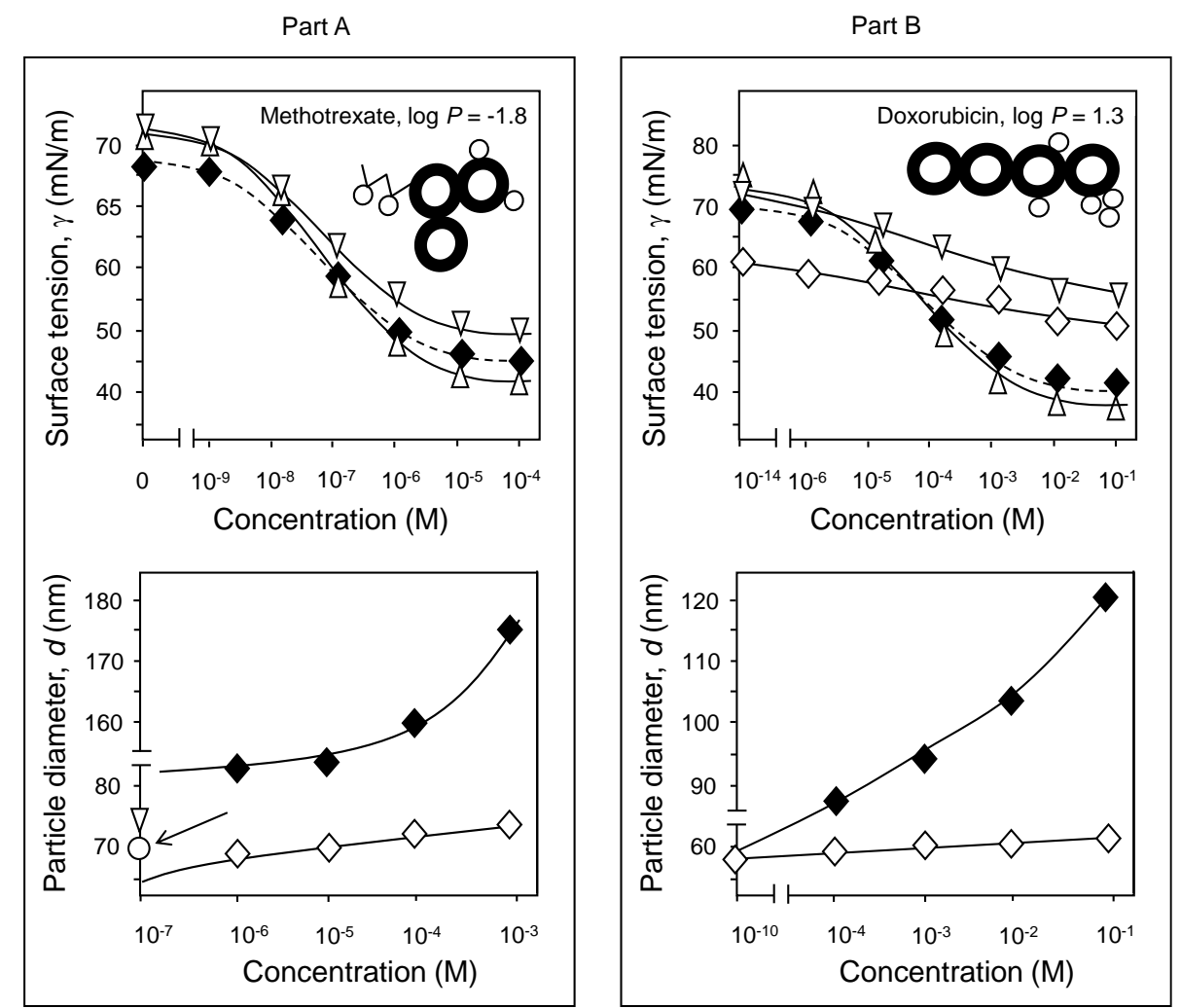

Fig. (12). Part A: shows the surface tension crossover for a mixed sample of increasing hydrophilic cationic cytotoxic drug methotrexate and with fixed $10^{-4} \mathrm{M}$ polymer Pluronic F68 $(\diamond)$ and F68 alone $(\Delta)$ in surface tension in the top plot for hydrophilic cytotoxic drug methotrexate $(\nabla)$, having a $\log P$ of -1.8 . The bottom panel of Part A, shows the increase in hydrodynamic diameter of liposomes of di-palmitoylphosphatidylcholine (DPPC) as determined by light scattering on inclusion of methotrexate $(\diamond)$, and a mixture involving $10^{-4}$ M Pluronic F68 and increasing concentrations of methotrexate $(\diamond)$. The diameters for bare DPPC liposomes $(\mathrm{O})$ and DPPC liposomes with F68 inclusion $(\nabla)$ are also represented in the lower scheme within part A. Part B: top panel shows the moderately hydrophobic but water dispersible $(\log P=1.3)$ cytotoxic drug, doxorubicin $(\nabla)$, surface active polymer Pluronic F108 $(\Delta)$, a mix of $10^{-4} \mathrm{M}$ doxorubicin with variable Pluronic F108 $(\diamond)$ and a mix of $10^{-4}$ M Pluronic F108 with variable doxorubicin $(\diamond)$. The bottom panel of Part $\mathrm{C}$ shows the increase in hydrodynamic diameter liposomes of di-palmitoylphosphatidylcholine (DPPC) as determined by light scattering on inclusion of increasing doxorubicin $(\diamond)$ and a mixture involving $10^{-2}$ M Pluronic F108 and increasing concentrations of doxorubicin $(\diamond)$. All solutions are made in $0.05 \mathrm{M}$ phosphate buffer, $\mathrm{pH} 7.0$ unless otherwise stated. 
points at inclusion of the poloxamer in the leaflet of the liposome and hydrophobic association of the doxorubicin with the poloxamer, in a similar fashion to the telodendrimer described by Jiang et al. [20] to give particles, which were double the diameter of liposomes with trace quantities of drug $[3,20,25,41,43]$ but possess the prerequisite hydrophilicity to aid long-acting therapeutic ability.

\section{FUTURE OPPORTUNITIES}

The faces of both the pharmaceutical sciences and of commercial therapeutic products, their working and diversity are changing toward the direction of smarter, purpose-designed fabrication. Increasingly complex versions of therapeutic products are needed to ensure their medical suitability, and this requires not only more careful consideration of the material itself but also the impact via complexation and surface adsorption of drugs, functional excipients and a range of combination products $[12,25$, $30,31,36,43,44]$ that possess these properties. Solids, macromolecules and simple surfactants in combination can all be used to functionalize bubble and droplet surfaces. A cartoon representation of the processes in action at the surface of drug delivery system containing nanoparticles and aggregates is shown phenomenologically in Fig. 13. The Figure attempts to summarize the diversity of surface forms seen in simple "protein-surfactant" combination systems (Figs. 3-5), and combination systems with bifunctional agents (Figs. 6-9), used as the basis of understanding less frequently studied drugsurfactant interactions [8, 10, 20, 43, 44, 51, 56], which show promise and significant interfacial behavior $[4,18,35,38,50]$ that can be utilized in various foam and emulsion systems. More importantly, a clearer understanding of pharmaceutical and vaccine dispersion interfacial properties, is made possible by consideration of better understood interfacial behavior (Figs. 3-8). Consequently, in the figures based on solid-in-water (S/W) drug dispersions (Figs. 11-12), with functional polymeric surfactants, liposomal phospholipids, silica [58] and licensed therapeutic molecules, it becomes possible to interpolate between the 'known' and the 'unknown', since identical physico-chemical principles drive the processes (overall behavior) occurring at the interface. Fig. 13 shows the interfacial structure of pharmaceutical foams, with their constituent bubble or gas cells and the emulsion droplets [19], typical of creams, lotions and parenteral injectable medicines. In the figure, a gross simplification, merely to aid the eye, means circles representing surfaceactive species. Shading of the circle points to the chemical form of the surface-active agent, which may be drug and possibly a surface-stabilizing agent or complex (or association structure) formed between the drug and stabilizer. Three combinations of any of these three amphiphiles is possible, these are lone (individual) molecules, a small cluster (aggregate) of interacted molecules or an entire network of interacted particles (Fig. 13), analogous to a twodimensional gel. The interaction can be diverse or simple, usually being complex, where proteins and hetero-polymers or drugs with multiple functional groups are concerned $[3,6,41,56]$. There is and has been considerable debate $[33,40,41]$ as to the form of such an adsorbed layer, which may change from a simple monolayer to multiple layers or strata, with varying degrees and depths of entanglement and interconnection (Fig. 2) of the primary and minor surface-constrained species. Previous studies with proteins, being based on many other findings, have largely indicated the presence of the latter for complex polymeric surfactants $[3,33,34,42,50]$. Simple monolayers are considered to be more prevalent with dispersions stabilized by smaller low molecular surfactants based on structural compositions of a simple head-group and aliphatic portions $[5,8,41$, 51, 54].

Areas currently challenging and exciting the physical science community in drug product development and drug-related physical chemistry (pharmaceutics), include those of, polymers for novel uses, novel biological sources $[14,31]$ but essentially with biocompatibility, controlled release of actives and environmental sensitivity or responsiveness. The latter is sometimes referred to as built-in intelligence or product smartness. This smartness is difficult to build-in because unlike many chemical engineering applications, medicine excipients must be non-toxic, yet functional. This means many past sources of the plethora of agents used for cosmetics, pharmaceuticals and vaccines include agents from food sources $[9,14,19,29$, $31,46,47]$ as primary or background components of these commodities could be useful. Models of liquid-liquid [19, 23, 55] and gas-liquid [5] interfaces explain the behavior well for similar simple pharmaceutical and medical products but are at odds with behavior in terms of complex simple or multi-phase coarse solid or crystalline particle suspensions; involving solid-water or solid-oil in- 
terfaces. This is because spatial confinement and restrictions to phase penetration, unlike water or oil continuous and dispersed phases [19], can be expected to mean differences in the polymer conformation. These are thus, unlike those of solidstate near-dry AFM measurements (Fig. 10), dissimilar to those measurements using an aqueous buffered medium for light scattering and $\zeta$ potential measurements (Figs. 11 and 12) but also arguably distinct from those using FRAP - probe mobility measurements (Figs. 7-8), interfacial rheology (Figs. 5, 7-9, 11) and interferometric measurements of foam film thickness (Fig. 6), exactly because of this confinement.

A starting point however, is needed and the array of techniques and disclosures (Fig. 1; Figs. 310) when combined do help to clarify what is important. They indicate which physico-chemical properties [38] are pivotal to function and which components can be manipulated for modification of interfacial properties and interfacial loading. One very important discovery has been the use of bifunctional agents (Figs. 6-9), which are able to unite regions of the adsorbed interfacial material $[14,27,32]$; these may be drug, excipient of background component as found in many vaccines. Consequently, promising additions for new forms of drug delivery and controlled release of actives (drugs) are now frequently used in state-of-the-art pharmaceutic and formulation developments. Such developments have included, use of polyphenols as antimicrobial agents [15], Pickering emulsions using solids [55], surface mechanical property 'switching' with peptides in emulsions [26] and defined aggregation number as in the case of telodendrimer encapsulation of cytotoxic drugs [20]. In addition, polymer-surfactant [4, 35, 43] and drug-surfactant complexes to aid drug delivery $[9,10,20,30,32,44,46,51]$ in colloidal and nano-scale structures, irreversibly adsorbed surfactant layers in emulsions [55], cationic surfactantstabilized organo-selenium nanoemulsions [24], hybrid foams based on emulsions [8, 25, 39] and cellulose nanofiber based stabilization of foams [7] also represent outstanding new developments in pharmaceutical physical chemistry. A mechanistic overview of why and how dispersions can be optimized is sometimes omitted in favor of therapeutic efficacy, however the two processes need not be mutually exclusive. Recent in-depth work does indeed concentrate on the molecular mechanisms in action and their mechanical manifestation with liquid-liquid, liquid-air and liquid-solid interfacial adsorbed material, including complexes and

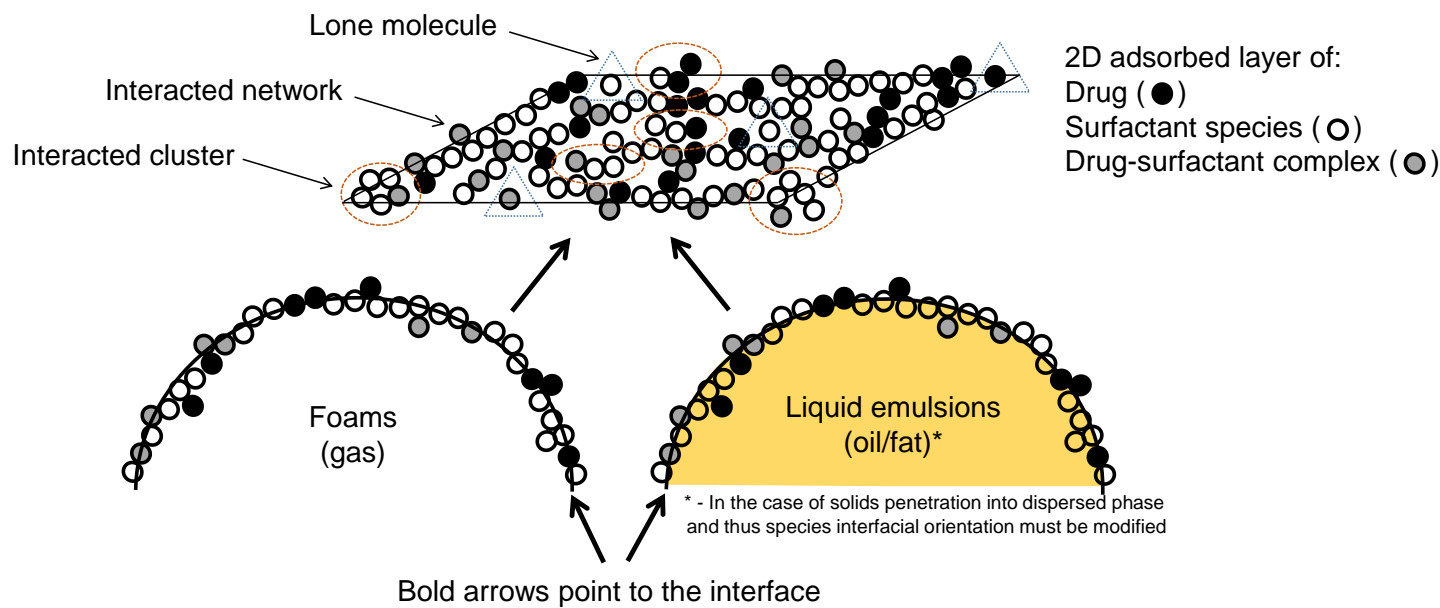

Fig. (13). The molecular interaction and freedom of lateral diffusion of species in the interfacial layer is represented phenomenologically. The mechanical properties and amount of associated solvent are entirely dictated by the profile of constituents of the adsorbed interfacial film of polymer, small molecule surfactant or drug species, which may be polymeric in nature or merely a simple amphiphile. The therapeutic entity entrained in the adsorbed film need not be surface active, such as simple ionic species, if it is complexed and bound to a species which shows surface active characteristics. Once all components are adsorbed at the surface there is capacity for a change in architecture based on the scope and range for a variety of interspecies interaction indicated in Fig. 2. Proteins and protein-based drugs are particularly powerful at forming intermolecular interaction between themselves because of the possibility of ionic, hydrogen, covalent, apolar and disulphide bridges and bonds. 
architectures $[19,20]$ involving therapeutic molecules $[10,21,39,43,46,51]$ of key medical significance.

\section{CONCLUSION}

A survey of past and present pharmaceutical science innovations and pragmatic drug delivery solutions shows it is both desirable and feasible to encapsulate drugs within solids, such as poly(caprolactone) beads and polymer micelles or hollow nanocapsules such as liposomes and nanoshells allowing both selective of differential containment and control over release. This miniature-scale and uniform distribution simultaneously aids therapeutic targeting and efficacy. Architectures and particles of this type have been used for some decades [2]. Recently, additional interest has been raised for the approach of surface functionalizing particles, such as latex beads, nanoemulsions, solid lipid nanoparticles and lipid nanocapsules for intravenous delivery. These entities are often referred to as nanomedicines because of their use of natural materials and thus, greater much improved biocompatibility over native nonmodified forms. Additionally, to a large extent and because of this modifying chemistry they have an increased chance of regulatory approval. Since there are already greater than 100 products of this type that have been created and approved (and many more in late stage clinical trials), new drug product filings in this class stand a greater chance of commercial release. Coarse dispersions (with 1$200 \mu \mathrm{m}$-sized dispersed phase components) such as, fine-cell foams, gels, suspensions and emulsions and related products, such as transdermal patch technologies, together with nanomedicines (approximately 50-150 nm-sized objects) represent a strategically valuable $20-30 \%$ of all medicinal products. This is despite solid dosage forms such as, tablets and capsules currently being the most common form of dosing a pharmaceutical (at approximately $60 \%$ of all drugs). This is not the case for vaccines however, since most vaccines are 'mixed systems' of oil, microbial lipid and solid material dispersed in a thickened emulsifier and 'protein-laden' aqueous dispersion, and are consequently, best thought of as low dispersed phase $\mathrm{O} / \mathrm{W}$ emulsions in the main. Solids and semisolids of the nanoparticle type used for intravenous delivery are to some extent more robust than coarse dispersions, particularly with respect to temperature and its sometimes disastrous effects on dispersed phase form. However, some drugs simply cannot be formulated in solid form because of poor aqueous solubility, potency issues and inherent toxicity and therefore, need to be formulated in colloidal or coarse dispersion form. All coarse dispersions due to their metastability suffer from difficulties of guaranteeing the microbiological safety and its assurance balanced against physico-chemical instability as a result of heavy hyperbaric or thermal processing.

New prospects for binding and housing high doses of drugs (a significant payload e.g. $70 \%$ by volume) not only within but also upon the surface of carrier vehicle particles means the potential for new gateways to the purposeful use of multiple therapeutics in 'cocktails' of encapsulated drugs. Alternatively, at the simplest level, to provide fully-loaded nanoparticle and coarse dispersion particles with a maximum amount of drug, which inturn would mean less frequent dosing. This is highly desirable for cytotoxic drugs in order to cut down cell apoptosis effects, such as tissue necrosis. It is not currently possible to exceed approximately $25-60 \%$ encapsulation ratios in dispersions for most drugs. Consequently, increased loading combined with complexation would provide the possibility for controlling the efflux of drug from the carrier and this would ensure safety and reduce side-effects. Pharmaceutical scientists are now fully aware of the opportunities for both modifying surface texture and increasing the encapsulation of drug in a more efficient manner. For coarse emulsions, such as anti-fungal and cortico-steroidal creams, modification of surface rheology had been shown to improve or extend shelf life by slowing down the rate and ease of coalescence [23] on droplet compaction or increased phase fluidity by thermal stressing and dispersed phase mobility in Ostwald ripening. Similar possibilities exist for pharmaceutical foams [12], although they are much less commonly used. However, dispersions of solids covered with functional polymers do present a huge opportunity for significant improvement to current practice and chances for revolutionary, life-changing new products $[27,56]$.

A fuller understanding, accrued from many of the fundamental and cross-disciplinary approaches of the combination of drug (bifunctional agent) and surfactants [17], and the role of complexes in surface functionalization will go a long way towards creating a new generation of products from pharma- 
ceutical scientists, to whom fabricating a better pharmaceutical and therapeutic product is now based on physics and physical chemistry. What sounds easy to invoke in a review, is actually much more difficult to put into practice. For example, many drugs are poorly water dispersible and so do not disperse in water in the first place, in order to make a foam or emulsion. Such hydrophobic particles tend to initiate rupture of the interstitial aqueous films or TLFs [2, 41] separating oil droplets, gas cells and solid particles [7, 27]. This happens because the particles are often poorly wettable and so have a large water contact angle. Hydrophilic drugs on the other hand, may possess marginal surfaceactivity and may also be devoid of the appropriate pendant functional groups to facilitate intermolecular interactions. From earlier work, and in appreciation of much current literature what seems difficult to engineer generically is the ratio of primary surface stabilizing agent and that of the active pharmaceutical ingredient or therapeutic species to the extent where surface rheology and texture is not compromised but where sufficient cavities and defects in the architecture $[19,20]$ and network have been created to allow a substantive incorporation of therapeutic. Finding the middle ground and the ideal situation is far from easy. This does become more facile when the drug itself is a bifunctional agent with the ability to attach to the primary surface functionalizer or is surface-active permitting coadsorption with the principal surfactant. This area of investigation is new, challenging and exciting with many opportunities for ingenious design and development in the fabrication of more useful therapeutics.

\section{CONSENT FOR PUBLICATION}

Not applicable.

\section{CONFLICT OF INTEREST}

The authors declare no conflict of interest, financial or otherwise.

\section{ACKNOWLEDGEMENTS}

DKS would like to thank Mike Helias, Dr Othman Al-Hanbali and Dr Gary Phillips from PABS at the University of Brighton for help in preparing samples for imaging and in obtaining the SEM and AFM images presented in Fig. 10.

\section{REFERENCES}

[1] Sarker DK. Quality Systems and Controls for Pharmaceuticals, John Wiley and Sons; 2008.

[2] Sarker DK. Pharmaceutical Emulsions: A Drug Developer's Toolbag, Wiley-Blackwell, 2013.

[3] Courthaudon J-L, Dickinson E. Competitive Adsorption of Lecithin and $\beta$-Casein in oil in Water Emulsions. J Agric Food Chem 1991; 39: $1365-8$.

[4] Pommier Y, Marchand C. Interfacial inhibitors: targeting macromolecular complexes. Nat Rev Drug Discov 2012; 11: 25-36.

[5] Shah V, Bharatiya B, Shukla, AD, Mukherjee T, Shah DO. Adsorption of non-ionic Brij and Tween surfactants at PTFE-water and air-water interfaces: Investigations on wetting, dispersion stability, foaming and drug solubilisation. Colloids Surfaces A: Physicochem Eng Aspects 2016; 508: 159-66.

[6] Kothur RR, Fucassi F, Dichello G, et al. Synthesis and applications of co-pillar[5]arene dithiols. Supramolecular Chem 2015; 28 (5-6): 436-43.

[7] Svagan AJ, Benjamins J-W, Al-Ansari Z, et al. Solid cellulose nanofiber based foams - Towards facile design of sustained drug delivery systems. J Controlled Release 2016; 244: 74-82.

[8] Sarker DK. Engineering of Nanoemulsions for Drug Delivery. Curr Drug Deliv 2005; 2 (4): 297-310.

[9] Puttipipatkhachorn S, Nunthianid J, Yamamoto K, Peck GE. Drug physical state and drug-polymer interaction on drug release from chitosan matrix films. J Controlled Release 2001; 75: 143-5.

[10] Mahajan S, Mahajan RK. Interactions of phenothiazine drugs with surfactants: A detailed physicochemical overview. Adv Colloid Interface Sci 2013; 199-200: 1-14.

[11] Valtcheva-Sarker RV, O'Reilly JD, Sarker DK. Administration of Drug and Nutritional Components in Nano-Engineered Form to Increase Delivery Ratio and Reduce Current Inefficient Practice. Recent Pat Drug Deliv Formul 2007; 1(2): 147-59.

[12] Arzhavitina A, Steckel, H. Foams for pharmaceutical and cosmetic application. Int J Pharm 2010; 394: 1-17.

[14] Kiszonas AM, Fuerst EP, Morris CF. Wheat Arabinoxylan Structure Provides Insight into Function. Cereal Chem 2013; 90(4): 387 95.

[15] Daglia M. Polyphenols as antimicrobial agents. Current Opinion Biotechnol 2012; 23(2):174-81.

[16] Kim K, Pack DW. In Lee A, Lee J (editors) In BioMEMS and Biomedical Nanotechnology, Vol. I Biological and Biomedical Nanotechnology. Springer; 2006. Chapter 2.

[17] $\mathrm{Li} \mathrm{Z}$, Tan $\mathrm{BH}$. Towards the development of polycaprolactone based amphiphilic block copolymers: molecular design, self-assembly and biomedical applications. Mater Sci Eng C 2014; 45: 620-34.

[18] Briceño-Ahumada Z, Soltero A, Maldonado A, Perez J, Langevin $\mathrm{D}$, Impéror-Clerc M. On the use of shear rheology to formulate stable foams. Example of a lyotropic lamellar phase. Colloids Surfaces A: Physicochem Eng Aspects 2016; 507: 110-17.

[19] Sun W, Sun D, Wei Y, Liu S, Zhang S. Oil-in-water emulsions stabilized by hydrophobically modified hydroxyethyl cellulose: Adsorption and thickening effect. J Colloid Interface Sci 2007; 311: 228-36.

[20] Jiang W, Wang X, Guo D, Luo J, Nangia S. Drug-Specific Design of Telodendrimer Architecture for Effective Doxorubicin Encapsulation. J Physical Chemistry B 2016; 120(36): 9766-77.

[21] Wieland DCF, Degen P, Paulus M, Schroer MA, Rehage H, Tolan M. $\mathrm{pH}$ controlled condensation of polysiloxane networks at the water-air interface. Colloids Surfaces A: Physicochem Eng Aspects 2014; 455: 44-8.

[22] Hu B, Wright RAE, Jiang S, Henn DM, Zhao B. Hybrid Micellar network hydrogels of thermosensitive ABA Triblock copolymer and polymer brush-grafted nanoparticles: Effect of LCST transition of polymer brushes on gel property. Polymer 2016; 82: 206-16.

[23] Reichert MD, Walker LM. Coalescence behaviour of oil droplets coated in irreversibly-adsorbed surfactant layers. J Colloid Interface Sci 2015; 449: 480-7.

[24] Piętka-Ottlik M, Lewińska A, Jaromin A, Krasowska A, Wilk KA. Antifungal organoselenium compound loaded nanoemulsions stabilized by bifunctional cationic surfactants. Colloids Surfaces A: Physicochem Eng Aspects 2016; 510: 53-62. 
[25] Zhao Y, Brown MB, Jones SA. Engineering novel topical foams using hydrofluroalkane emulsions stabilised with Pluronic surfactants. Eur J Pharm Sci, 2009; 37: 370-7.

[26] Dexter AF, Malcolm AS, Middelberg APJ. Reversible active switching of the mechanical properties of a peptide film at a fluidfluid interface. Nature Mat 2006; 5: 502-6.

[27] Sarker DK, Wilde PJ, Clark DC. Enhancement of the stability of protein-based foams using trivalent cations. Colloids Surfaces A: Physicochem Eng Aspects 1996; 14: 227-36.

[28] Dimitrova LM, Boneva MP, Danov KD, et al. Limited coalescence and Ostwald ripening in emulsions stabilized by hydrophobin HFBII and milk proteins. Colloids Surfaces A: Physicochem Eng Aspects 2016; 509: 521-38.

[29] Singh AV, Bandgar BM, Kasture M, Prasad BLV, Sastry M. Synthesis of gold, silver and their alloy nanoparticles using bovine serum albumin as foaming and stabilizing agent. J Mater Chem 2005;15: 5115-21.

[30] Gao Z-M, Wang J-M, Wu N-N, et al. Formation of Complex Interface and Stability of Oil-in-Water $(\mathrm{O} / \mathrm{W})$ Emulsion Prepared by Soy Lipophilic Protein Nanoparticles. J Agricultural Food Chem 2013; 61(32): 7838-47.

[31] Lam S, Velikov KP, Velev OD. Pickering stabilization of foams and emulsions with particles of biological origin. Curr Opinion Colloid Interface Sci 2014; 19: 490-500.

[32] Littoz F, McClements DJ. Bio-mimetic approach to improving emulsion stability: Cross-linking adsorbed beet pectin layers using laccase. Food Hydrocolloids 2008; 22: 1203-11.

[33] Borbás R, Murray BS, Kiss É. Interfacial shear rheological behaviour of proteins in three-phase partitioning systems. Colloids Surfaces A: Physicochem Eng Aspects 2003; 213(1): 91-103.

[34] Deshmukh OS, van den Ende D, Stuart MC, Mugele F, Duits MHG. Hard and soft colloids at fluid interfaces: Adsorption, interactions, assembly \& rheology. Adv Colloid Interface Sci 2015; 222: $215-27$

[35] Qi S, Roser S, Edler KJ, et al. Insights into the Role of PolymerSurfactant Complexes in Drug Solubilisation/Stabilisation During Drug Release from Solid Dispersions. Pharm Res 2013; 30(1): 290302 .

[36] Li W, Huang Z, Wu Y, Zhao X, Liu S. Honeycomb carbon foams with tunable pore structures prepared from liquefied larch sawdust by self-foaming. Ind Crops Prod 2015; 64: 215-23.

[37] Rio E, Drenckhan W, Salonen A, Langevin D. Unusually stable liquid foams. Adv Colloid Interface Sci 2014; 205: 74-86.

[38] Schreier S, Malheiros SVP, de Paula E. Surface active drugs: Selfassociation and interaction with membranes and surfactants. Physicochemical and biological aspects. Biochimica Biophysica Acta (BBA) - Biomembranes 2000; 1508(1): 210-34

[39] Gao Z, Zhan W, Guo Y, Wang Y, Guo Y, Lu G. Aldehydepropylfunctionalized mesostructured cellular foams: Efficient supports for immobilization of penicillin G acylase. J Molecular Catalysis B: Enzymatic 2014; 105: 111-17.

[40] Wüstneck R, Krägel J, Miller R, et al. Dynamic surface tension and adsorption properties of beta-casein and beta-lactoglobulin. Food Hydrocolloids 1996; 10(4), 395-405.

[41] Sarker DK, Wilde PJ, Clark DC. Competitive adsorption of L- $\square-$ lysophosphatidylcholine/ $\beta$-lactoglobulin mixtures at the interfaces of foams and foam lamellae. Colloids Surfaces B: Biointerfaces 1995; 3: 349-56.

[42] Dickinson E, Golding M. Rheology of Sodium Caseinate Stabilized Oil-in-Water Emulsions. J Colloid Interface Sci 1997; 191: 166-76.
[43] Akpalo E, Bidault L, Boissière M, Vancaeyzeele C, Fichet O, Larreta-Garde V. Fibrin-polyethylene oxide interpenetrating polymer networks: New self-supported biomaterials combining the properties of both protein gel and synthetic polymer. Acta Biomaterialia 2011; 7(6): 2418-27.

[44] García MC, Cuggino JC, Rosset C, et al. A novel gel based on an ionic complex from a dendronized polymer and ciprofloxacin: Evaluation of its use for controlled topical drug release. Mater Sci Eng C 2016; 69: 236-46.

[45] Niang PM, Huang Z, Dulong V, Souguir Z, Le Cerf D, Picton L. Thermo-controlled rheology of electro-assembled polyanionic polysaccharide (alginate) and polycationic thermo-sensitive polymers. Carbohydr Polym 2016; 139: 67-74.

[46] Streck L, de Araújo MM, de Souza I, et al. Surfactant-cosurfactant interactions and process parameters involved in the formulation of stable and small droplet-sized benznidazole-loaded soybean $\mathrm{O} / \mathrm{W}$ emulsions. J Mol Liq 2014; 196: 178-86.

[47] Alhaique F, Casadei MA, Cencetti C, et al. From macro to nano polysaccharide hydrogels: An opportunity for the delivery of drugs. J Drug Deliv Sci Technol 2016; 32: 88-99.

[48] Shah A, Masoodi FA, Gani A, Ashwar BA. In-vitro digestibility, rheology, structure, and functionality of RS3 from oat starch. Food Chem 2016; 212: 749-58.

[49] Shulze M, Handge UA, Rangou S, Lillepärg J, Abetz V. Thermal properties, rheology and foams of polystyrene-block-poly(4vinylpyridine) diblock copolymers. Polymer 2015; 70: 88-99.

[50] Ma Q, Du L, Yang Y, Wang L. Rheology of film-forming solutions and physical properties of Tara gum film reinforced with polyvinyl alcohol (PVA). Food Hydrocolloids 2017; 63: 677-84.

[51] Sharma R, Nandni D, Mahajan RK. Interfacial and micellar properties of mixed systems of tricyclic antidepressant drugs with polyoxyethylene alkyl ether surfactants. Colloids Surfaces A: Physicochem Eng Aspects 2014; 451: 107-16.

[52] Fan J, Liu F, Wang Z. Shear rheology and in-vitro release kinetic study of apigenin from lyotropic liquid crystal. Int J Pharm 2016; 497(1-2): 248-54.

[53] Gavara N, Chadwick RS. Noncontact microrheology at acoustic frequencies using frequency-modulated atomic force microscopy. Nature Methods 2010; 7: 650-4.

[54] Al-Hanbali O, Onwuzo NM, Rutt K, Dadswell CM, Moghimi SM, Hunter AC. Modification of the Stewart biphasic colorimetric assay for stable and accurate quantitative determination of Pluronic and Tetronic block copolymers for application in biological systems. Anal Biochem 2007; 361 (2): 287-93.

[55] Chevalier Y, Bolzinger M-A. Emulsions stabilized with solid nanoparticles: Pickering emulsions. Colloids Surfaces A: Physicochem Eng Aspects 2013; 439: 23-34.

[56] Bazylińska U, Zieliński W, Kulbacka J, Samoć M, Wilk KA. New diamidequat-type surfactants in fabrication of long-sustained theranostic nanocapsules: Colloidal stability, drug delivery and bioimaging. Colloids Surfaces B: Biointerfaces 2016; 137: 121-32.

[57] Čop M, Lacoste C, Conradi M, Laborie M-P, Pizzi A, Sernek M. The effect of the composition of spruce and pine tannin-based foams on their physical, morphological and compression properties. Ind Crops Prod 2015; 74: 158-64.

[58] Thomas PC, Cipriano BH, Raghavan SR. Nanoparticle-crosslinked hydrogels as a class of efficient materials for separation and ion exchange. Soft Matter 2011; 7: 8192-7. 